\title{
Log-gamma directed polymer with one free end via coordinate Bethe Ansatz
}

\author{
Pascal Grange \\ Department of Mathematical Sciences \\ Xi'an Jiaotong-Liverpool University \\ 111 Ren'ai Rd, 215123 Suzhou, China \\ pascal.grange@xjtlu.edu.cn
}

\begin{abstract}
The discrete polymer model with random Boltzmann weights with homogeneous inverse gamma distribution, introduced by Seppäläinen, is studied in the case of a polymer with one fixed and one free end. The model with two fixed ends has been integrated by Thiery and Le Doussal, using coordinate Bethe Ansatz techniques and an analytic-continuation prescription. The probability distribution of the free energy has been obtained through the replica method, even though the moments of the partition sum do not exist at all orders due to the fat tail in the distribution of Boltzmann weights. To extend this approach to the polymer with one free end, we argue that the contribution to the partition sums in the thermodynamic limit is localised on parity-invariant string states. This situation is analogous to the case of the continuum polymer with one free end, related to the Kardar-Parisi-Zhang equation with flat boundary conditions and solved by Le Doussal and Calabrese. The expansion of the generating function of the partition sum in terms of numbers of strings can also be transposed to the log-gamma polymer model, with the induced Fredholm determinant structure. We derive the large-time limit of the rescaled cumulative distribution function, and relate it to the GOE Tracy-Widom distribution. The derivation is conjectural in the sense that it assumes completeness of a family of string states, and expressions of their norms, already useful in the fixed-end problem, and extends heuristically the order of moments of the partition sum to the complex plane.
\end{abstract}




\section{Contents}

1 Introduction and conclusions 2

2 Review and quantities of interest 5

2.1 Definition of the model . . . . . . . . . . . . . . . . . 5

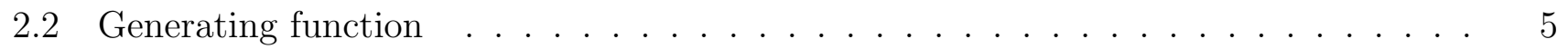

2.3 Divergences and analytic continuation of the moments . . . . . . . . . . 6

2.4 Time-evolution of moments . . . . . . . . . . . . . . . . . 7

2.5 String states in the thermodynamic limit . . . . . . . . . . . . . . . . 9

2.6 Expansion of the generating function in terms of strings . . . . . . . . . . . 10

3 Localisation on parity-invariant string states $\quad 11$

3.1 Symmetry argument from the overlap factor . . . . . . . . . . . . . . . 11

3.2 Time-evolution factors of parity-invariant string states . . . . . . . . . . . . 12

3.3 Norm of the parity-invariant states and overlap with the uniform wave function . . 12

3.3.1 Norms of the string states of the log-gamma model . . . . . . . . . . . 12

3.3.2 Form of the overlaps . . . . . . . . . . . . . . . . 14

3.4 Measure-theoretic factors . . . . . . . . . . . . . . . . . . 15

3.4.1 Pairs of strings with identical number of particles and opposite linear momenta 16

3.4 .2 One string with zero momentum . . . . . . . . . . . . . . 17

4 The one-string contribution to the generating function at large time 18

\begin{tabular}{lll}
5 & Pfaffian structure of the generating function & 20 \\
\hline
\end{tabular}

6 The two-string contribution to the generating function at large time 23

6.1 One pair of strings with identical numbers of particles . . . . . . . . . . . 23

6.2 Two parity-invariant strings with independent numbers of particles . . . . . . . 25

7 Numerical tests

\section{Introduction and conclusions}

The application of the methods of integrable systems to the continuum Kardar-Parisi-Zhang (KPZ) equation [1, 2], a model of one-dimensional growth of an interface in the presence of noise, led to an exact solution. In particular, the height field of the interface was characterised by its probability density, by means of the Cole-Hopf mapping which relates the height field to the free energy of a directed-polymer model. The time evolution of the integer moments of the partition sum of this model, in the replica approach, was mapped to the one-dimensional Lieb-Liniger model of interacting bosons [3 5], which is solvable by Bethe Ansatz methods [6]. The collection of exact expressions for these moments yielded the probability density of the rescaled free energy for the most studied classes of boundary conditions for the KPZ equation. The moments were rewritten in terms of the Airy function, as they grow proportionally the exponential of the cube of their order. The resulting kernels allowed to relate the probability density of the free energy at large times to 
the Tracy-Widom [7,8] distributions of the largest eigenvalue of large Gaussian random matrices. Moreover, the generating function of moments was expressed as a Fredholm determinant for several classes of boundary conditions.

While this research program has been completed for continuum models, yielding insights on the KPZ universality class $[11,32$, an alternative approach is the study of discrete models of directed polymers (or integrable particle systems or growth models), whose solutions can yield results for the continuum model by taking a suitable limit (see [33] for a recent review, and [34 41] for more families of models and their classification, and [42,43 for rigorous solutions involving the replica approach). In particular, the log-gamma polymer model introduced by Seppäläinen [44], defined by the distribution of Boltzmann weights on a lattice (with a fat-tailed distribution of weights characterised by an exponent denoted by $\gamma$ ), was solved by combinatorial methods [45], and the generating function was related to a Fredholm determinant [46].

On the other hand, a crucial step towards the extension of the Bethe Ansatz replica approach to discrete models was taken by Thiery and Le Doussal in [47], where the fixed-end log-gamma model was solved using the coordinate Bethe Ansatz approach due to Brunet, yielding eigenfunctions of the replica transfer matrix. In particular, the tangents of the rapidities, which are used to express these eigenfunctions, are regularly spaced on segments of the complex plane in the thermodynamic limit. This reproduces the so-called string structure that rapidities exhibit in the Bethe Ansatz solution of the Lieb-Liniger model.

However, the fat tail of the distribution of Boltzmann weights is an obstacle to the replica approach to the log-gamma polymer, which makes use the moments of the partition sum at all orders, whereas these moments diverge at orders larger than the parameter $\gamma$. An analytic-continuation prescription inspired by the Mellin representation of the exponential function was given in [47] to express the generating function, given a formal expression of integer moments of the partition sum. This led to an expression of the generating function as a Fredholm determinant. At large times the rescaled cumulative distribution function of the free energy of the log-gamma directed polymer with fixed ends, reduces to the Gaussian Unitary Ensemble (GUE) Tracy-Widom distribution. This completed the replica Bethe Ansatz program for the log-gamma polymer with fixed ends, whose continuum limit can be related to the KPZ problem with droplet boundary conditions. This paper aims to extend this approach to the large-time limit of the log-gamma polymer with one free end and one fixed end (whose continuum limit corresponds to the KPZ problem with flat boundary conditions). The results are conjectural in the sense that the same assumptions are made as in [47], including the completeness of the string solutions in the thermodynamic limit, the expression of the norms of these states, and the analytic continuation to complex values of the number of replicas. Moreover, the symmetry argument used to restrict the calculation to parity-invariant states is only supported by checks in special cases.

The outline of the paper is as follows. In Section 2 the log-gamma polymer model is reviewed. Notations are introduced for the rescaled free energy, and the analytic-continuation prescription is motivated by the divergence of the moments at time zero, which occurs in the free-end case just as in the fixed-end case. The partition sum of the free-end model takes the form of a sum over all the fixed-end sectors. Expressing the moments in terms of the orthogonal Bethe eigenfunctions 
(assumed to form a complete set of states) yields a sum of quantities known from the fixed-end solution of [47], weighted by overlaps between eigenfunctions and a uniform wave function on the lattice. These overlaps are therefore the only quantities left to compute. In Section 3 we express these overlaps after arguing that the phase-space integration localises on parity-invariant systems of string states. String states appeared crucially for the continuum polymer model with one free end in the derivation of Calabrese and Le Doussal in [14, 15], and were reobtained and interpreted by means of a symmetry argument in [31] based on the results of [49] involving half-strings. The fact that the string states in the thermodynamic limit of the log-gamma model are obtained from the rapidities by an odd mapping relates the two situations. In Section 4 we work out the first term in the string expansion of the generating function, which induces the centering and scaling of the free energy in terms of the parameter $\gamma$. After analytic continuation, the large-time limit of this term is studied using a saddle-point method and the Airy function, and found to be independent of the parameter $\gamma$. Numerical checks are reported in the final section, based on direct simulations of the system with $10^{4}$ samples, at time $t=4,096$, and for $\gamma=3$ (a value for which the divergence of moments is especially severe). It should be noted that an alternative (rigorous, non-asymptotic) approach was recently taken in [48], unifying classes of boundary conditions at finite time through classes of Whittaker functions, including the one considered in the present paper. The classification of stochastic particle systems with zero-range interaction [34] induces integrability conditions on classes of directed-polymer models in random environment [35], including the beta and inversebeta polymers. These models are related by a web of dualities, and can exhibit features including anisotropy, degeneration to the log-gamma model in certain limits, and convergence of moments. They could provide natural ground for applications of the techniques developed in the present work.

The full string expansion of the generating function is arranged into a Fredholm Pfaffian, using identities that reproduce the algebraic structure of the derivation in the continuum polymer model [15], once the appropriate kernels are known for the discrete log-gamma model. The large-time limit of this Fredholm Pfaffian yields the characterisation of the cumulative distribution function of the (centered and rescaled) free energy of the log-gamma polymer with one free end, in terms of the Tracy-Widom distribution:

$$
\lim _{t \rightarrow \infty} \operatorname{Prob}\left(\frac{\left(\log Z_{t}+\psi\left(\frac{\gamma}{2}\right) t\right)}{\left(-\frac{t}{8} \psi^{\prime \prime}\left(\frac{\gamma}{2}\right)\right)^{\frac{1}{3}}}<s\right)=\sum_{n \geq 0} \frac{1}{n !} z(n, s),
$$

where $\psi$ is the digamma function. The integer parameter $n$ is the number of elements in the string expansion, and for each value of $n$ the $n$-string contribution reads

$$
z(n, s)=(-1)^{n} \int_{\mathbf{R}^{n}}\left(\prod_{k=1}^{n} d x_{k}\right) \operatorname{det}\left[B_{s}\left(x_{i}, x_{j}\right)\right]_{n \times n},
$$

and the kernel $B_{s}$ is defined in terms of the Airy function as

$$
B_{s}(x, y)=\theta(x) \operatorname{Ai}(x+y+s) \theta(y),
$$

where $\theta(x)=\mathbf{1}(x>0)$, and the r.h.s. of Eq. 1 is the Gaussian Orthogonal Ensemble (GOE) Tracy-Widom distribution, denoted by $F_{1}(s)$. This universal function also governs the distribution function of the large-time limit of the continuum polymer model with one free end, mapped to the KPZ equation with flat boundary conditions. 


\section{Review and quantities of interest}

\subsection{Definition of the model}

Given a rectangular lattice with vertices labeled by the discrete values of the coordinates $x$ and $t$, random Boltzmann weights denoted by $w_{x, t}$ attached to each vertex. In the log-gamma directed polymer model [44], these weights (at finite temperature set to unity) are independent identicallydistributed according to the distribution:

$$
P_{\gamma}(w) d w=\frac{1}{\Gamma(\gamma)} w^{-1-\gamma} e^{-1 / w} d w
$$

with fixed parameter $\gamma>0$.

The discrete one-dimensional log-gamma directed polymer is decribed with half-integer space coordinate $x$ and integer time coordinate $t$, starting at the fixed end $(x=0, t=0)$, follows paths with the constraint allowing only jumps from $(x, t)$ are to $\left(x \pm \frac{1}{2}, t+1\right)$. The allowed values of the coordinate $x$ at even (resp. odd) times are therefore integer (resp. half-odd) numbers. The allowed paths are therefore equivalent to up-right directed paths on a two-dimensional lattice with integer coordinates $i, j$, with starting point at $(i=1, j=1)$ and the relations $t=i+j-2$ and $x=(i-j) / 2$.

Boltzmann weights are multiplicative along paths: a random quantity can be associated to a path on the lattice by taking the product of the random weights along the path. At time $t$, the partition function $Z_{t}$ of the directed polymer starting with a specified starting point, say at $x=0$, and one free end, is defined as the sum of the Boltzmann weights of the paths $\phi$ with $t$ time steps and the specified starting point:

$$
Z_{t}:=\sum_{x \in\{0, \ldots, L-1\}} \sum_{\phi:(0,0) \rightarrow(x, t)} \prod_{\left(x^{\prime}, t^{\prime}\right) \in \phi} w_{x^{\prime}, t^{\prime}}
$$

where $L$ is the number of sites in one dimension. Moreover, at any value of time, the partition function of the polymer with one free end is the sum of the partition function $Z_{t}^{f i x e d}$ of the polymer with two fixed ends (one at 0 , one at $x$ ), over the possible values of the final position:

$$
Z_{t}^{\text {fixed }}(x):=\sum_{\phi:(0,0) \rightarrow(x, t)} \prod_{\left(x^{\prime}, t^{\prime}\right) \in \phi} w_{x^{\prime}, t^{\prime}}
$$

which was studied in 4547 .

\subsection{Generating function}

With the aim of characterising the probability distribution function of the free energy of the loggamma polymer with one free end, let us consider the $\lambda$-dependent generating function 1 .

$$
g_{\lambda}(s)=\overline{\exp \left(-e^{-\lambda s} Z_{t}\right)}
$$

\footnotetext{
${ }^{1}$ We will sometimes drop the index $t$ in notations involving the the partition sum.
} 
where the overline denotes the average over the disorder introduced by the random nature of the Boltzmann weights, described by Eq. 4. With the definition of the rescaled free energy $f$

$$
-\log Z_{t}=: \lambda f
$$

where the value of $\lambda$ is assumed to scale as a growing function of time so that the large-time limit of the probability distribution of $f$ exists (and this limit can be captured by the large- $\lambda$ limit, see Section 4), the generating function becomes

$$
g_{\lambda}(s)=\overline{\exp \left(-e^{-\lambda(s+f)}\right)}
$$

so that the large- $\lambda$ limit of the expression to be averaged under the disorder is just a step function, and the large- $\lambda$ limit of the generating function is related to the cumulative distribution function of $f$ :

$$
\lim _{\lambda \rightarrow \infty} g_{\lambda}(s)=\overline{\mathbf{1}(f+s>0)}=\int_{-s}^{+\infty} P(f) d f,
$$

from which we see that the probability distribution function of $f$ can be computed from the large- $\lambda$ of the generating function.

Expanding the generating function in powers of $Z_{t}$ in the definition of Eq. 7, and taking the average over disorder of each term yields the expression

$$
g_{\lambda}^{m o m}(s):=1+\sum_{n \geq 1} \frac{\overline{Z_{t}^{n}}}{n !}(-1)^{n} e^{-\lambda n s},
$$

from which we see that computing the moments $\overline{Z_{t}^{n}}$ at all orders $n$ is enough to characterise $g_{\lambda}^{\text {mom }}$. Moreover, the parameter $\lambda$ can be thought of as a chemical potential associated to the number of particles $n$. However, the moments of the log-gamma model suffer from divergences at sufficiently large orders, so that the expression of $g_{\lambda}^{\text {mom }}$ is only formal approach to the calculation of the generating function.

\subsection{Divergences and analytic continuation of the moments}

The partition function $Z_{0}$ is the same as in the case of the polymer with fixed ends, as both models admit just one point-like path at time $t=0$. Its moment of order $n$ is expressed as

$$
\overline{Z_{0}^{n}}=\overline{w^{n}}=\frac{1}{\Gamma(\gamma)} \int_{0}^{\infty} w^{-1+n-\gamma} e^{-1 / w} d w=\frac{\Gamma(\gamma-n)}{\Gamma(\gamma)}
$$

This moment does not exist as an integral for $n$ larger than the parameter $\gamma$. However, its expression can be analytically continued to all complex values $n$ (except integers large enough to give rise to poles in the Gamma function). Following Appendix A of [47], the generating function can therefore be conjectured to be obtainable from formal sums of moments by an analytic-continuation 
prescription. The Mellin representation of the exponential function, which can be rewritten using the Euler reflection formula as follows:

$$
e^{-z}=\int_{-a+i \mathbf{R}} \frac{d m}{2 i \pi} \Gamma(-m) z^{m}=-\int_{-a+i \mathbf{R}} \frac{d m}{2 i \sin (\pi m)} \frac{1}{\Gamma(1+m)} z^{m},
$$

where $a>0$, allows one to express the generating function as an integral:

$$
\begin{aligned}
g_{\lambda}(s) & =\overline{\exp \left(e^{-\lambda s} Z\right)} \\
& =-\int P(Z)\left(\int_{-a+i \mathbf{R}} \frac{d m}{2 i \sin (\pi m)} \frac{1}{\Gamma(1+m)} e^{-\lambda s m} Z^{m}\right) d Z \\
& =\int_{-a+i \mathbf{R}} \frac{d m}{2 i \sin (\pi m)} \frac{1}{\Gamma(1+m)} e^{-\lambda s m} \overline{Z^{m}} .
\end{aligned}
$$

After obtaining expressions for integer moments, formal sums such as $g_{\lambda}^{m o m}$ will eventually be related to the generating function using continuation to complex values of $m$ with the integration prescription of Eq. 14 .

\subsection{Time-evolution of moments}

The discrete-time evolution of each path induces the following time-evolution equation for the partition function with fixed end:

$$
Z_{t+1}^{\text {fixed }}(x)=w_{x, t+1}\left(Z_{t}^{\text {fixed }}\left(x-\frac{1}{2}\right)+Z_{t}^{\text {fixed }}\left(x+\frac{1}{2}\right)\right)
$$

and boundary condition $Z_{0}^{\text {fixed }}(x)=w_{0,0} \delta_{x, 0}$. Let us review the analogy worked out in Section 3 of [47] between this problem and the Schrödinger equation (with the Lieb-Liniger Hamiltonian [3]) that is known to govern the time-evolution of the moments in the continuum case.

At a given order $n$, if the role of the wave function at time $t$ in the discrete setting of the log-gamma polymer is played by $\psi_{t}\left(x_{1}, \ldots, x_{n}\right)$ defined in terms of the moments of the fixed-end partition sum by

$$
\overline{\prod_{i=1}^{n} Z_{t}^{\text {fixed }}\left(x_{i}\right)}=: 2^{n t}\left(\frac{\bar{c}}{4}\right)^{n(t+1)} \psi_{t}\left(x_{1}, \ldots, x_{n}\right)
$$

and

$$
\bar{c}=\frac{4}{\gamma-1}
$$

the time-evolution equation of $Z_{t}^{\text {fixed }}$ induces the following linear time-evolution equation, which motivates the definition of the operator $H_{n}$ (which is a discrete analogue of the Lieb-Liniger Hamiltonian of the quantum mechanics of bosons on a line), which we quote from [47]:

$$
\psi_{t+1}\left(x_{1}, \ldots, x_{n}\right)=\left(\frac{1}{2^{n}} a_{x_{1}, \ldots, x_{n}}\right) \sum_{\left(\delta_{1}, \ldots, \delta_{n}\right) \in\left\{-\frac{1}{2}, \frac{1}{2}\right\}^{n}} \psi_{t}\left(x_{1}-\delta_{1}, \ldots, x_{n}-\delta_{n}\right)=:\left(H_{n} \psi_{t}\right)\left(x_{1}, \ldots, x_{n}\right),
$$


with the notation

$$
a_{x_{1}, \ldots, x_{n}}=(\gamma-1)^{n} \overline{\prod_{\alpha=1}^{n} w_{x_{\alpha}, t+1}}=\prod_{x} h_{\sum_{\alpha=1}^{n} \delta_{x, x_{\alpha}}}, \quad \text { and } \quad h_{n}=\prod_{k=0}^{n-1} \frac{4}{4-k \bar{c}} .
$$

Consider an orthogonal set of normalisable eigenstates, denoted by $|\mu\rangle$ (labeled by an index $\mu$ ), of the time-independent Schrödinger equation associated to the operator $H_{n}$ for a fixed number $n$ of points, with associated wave functions $\Psi_{\mu}$, corresponding to the eigenvalues $\theta_{\mu}$. This set is assumed to be complet $\varrho^{2}$, so that for all values of the index $\mu$ labeling the set of states,

$$
H_{n} \Psi_{\mu}\left(x_{1}, \ldots, x_{n}\right)=\theta_{\mu} \Psi_{\mu}\left(x_{1}, \ldots, x_{n}\right)
$$

From the definition of the partition function of the free-end model (Eq. 6), we can see that the moment of order $n$ of the partition function $Z_{t}$ of the model with one free end is given by a multiple sum (over the coordinates of the fixed end) of the moment of order $n$ the partition function with fixed ends:

$$
\overline{Z_{t}^{n}}=\sum_{\left(x_{1}, \ldots, x_{n}\right) \in\{0, \ldots, L-1\}} \overline{\prod_{i=1}^{n} Z_{t}^{\text {fixed }}\left(x_{i}\right)}
$$

The time-evolution of the moment $\overline{Z_{t}^{n}}$ can therefore be worked out using the eigenvalues of the Hamiltonian $H_{n}$, and projecting the initial value of the moment of the fixed-end model onto the eigenstates of the Hamiltonian (using the completeness assumption):

$$
\left|\psi_{0}\right\rangle=\sum_{\mu} \frac{\left\langle\mu \mid \psi_{0}\right\rangle}{\langle\mu \mid \mu\rangle}|\mu\rangle, \quad\left|\psi_{t}\right\rangle=\sum_{\mu} \theta_{\mu}^{t} \frac{\left\langle\mu \mid \psi_{0}\right\rangle}{\langle\mu \mid \mu\rangle}|\mu\rangle
$$

Using Eq. 16 yields

$$
\overline{\prod_{i=1}^{n} Z_{t}^{\text {fixed }}\left(x_{i}\right)}=2^{n t}\left(\frac{\bar{c}}{4}\right)^{n(t+1)} \sum_{\mu} \theta_{\mu}^{t} \frac{\left\langle\mu \mid \psi_{0}\right\rangle}{\langle\mu \mid \mu\rangle} \Psi_{\mu}\left(x_{1}, \ldots, x_{n}\right)
$$

which was calculated in [47]. Upon permuting the sum over states and the sum over coordinates of the free ends of the polymers, we obtain

$$
\overline{Z_{t}^{n}}=2^{n t}\left(\frac{\bar{c}}{4}\right)^{n(t+1)} \sum_{\mu} \theta_{\mu}^{t} \frac{\left\langle\mu \mid \psi_{0}\right\rangle}{\langle\mu \mid \mu\rangle}\left(\sum_{x_{1}, \ldots, x_{n} \in\{0, \ldots, L-1\}} \Psi_{\mu}\left(x_{1}, \ldots, x_{n}\right)\right),
$$

from which we observe that the only quantities left to compute once the solution to the fixed-end problem is known are the overlaps

$$
\Omega_{\mu}:=\sum_{x_{1}, \ldots, x_{n} \in\{0, \ldots, L-1\}} \Psi_{\mu}\left(x_{1}, \ldots, x_{n}\right)
$$

\footnotetext{
${ }^{2}$ This set will eventually be taken to be indexed by the systems of strings worked out in $[47$ in the thermodynamic limit, together with the eigenvalues, and shown to be orthogonal with respect to a weighted scalar product depending on the log-gamma distribution, whose explicit expression (given in Section 4.1 of [47]) does not need to be reproduced here, as we will only use the orthogonality property rather than the explicit form of the product.
} 
of the eigenstates with the uniform function over the discretized $n$-dimensional space, which are the discrete analogs of the integrals worked out in [15] and reobtained in 31] through a symmetry argument to solve the KPZ problem with flat boundary conditions. We therefore have to review the set of eigenstates labeled by $\mu$ used in [47] to solve the fixed-end log-gamma model in the thermodynamic limit.

\subsection{String states in the thermodynamic limit}

Eigenfunctions of the operator $H_{n}$ can be expressed by coordinate Bethe Ansatz techniques discovered by Brunet. They are symmetrized superpositions of plane waves, corresponding to the rapidities $\left(\lambda_{\alpha}\right)_{1 \leq \alpha \leq n}$ :

$$
\Psi_{\mu}\left(x_{1}, \ldots, x_{n}\right)=\sum_{\sigma \in \mathcal{S}_{n}} A_{\sigma} \prod_{i=1}^{n} e^{i \lambda_{\sigma(\alpha)} x_{\alpha}}
$$

where

$$
A_{\sigma}=\prod_{1 \leq \alpha<\beta \leq n}\left(1+\frac{\bar{c}}{2} \frac{\operatorname{sign}\left(x_{\beta}-x_{\alpha}+0^{+}\right)}{t_{\sigma(\alpha)}-t_{\sigma(\beta)}}\right)
$$

with the notation

$$
t_{\alpha}=i \tan \left(\frac{\lambda_{\alpha}}{2}\right)
$$

which implies that the plane waves can be expressed in terms of the family of parameters $\left(t_{\alpha}\right)_{1 \leq \alpha \leq n}$ :

$$
z_{\alpha}=e^{i \lambda_{\alpha}}=\frac{1+t_{\alpha}}{1-t_{\alpha}}
$$

The rapidities are names by analogy with the usual coordinate Bethe Ansatz [6], much of the structure of which can be recognised in Eq. 26, up to the fact that the role played in the combinatorial factors by $\lambda_{\alpha}$ in the Bethe Ansatz is played by $t_{\alpha}$ (up to a factor of $2 i$ ) in the Ansatz of Eq. 26.

In the special case $n=2$, working out the eigenvalues $\theta_{\mu}$ in the diagonalisation problem of Eq. 20 yields $\theta_{\mu,(n=2)}=\frac{1}{4} \sum_{\alpha, \beta \in\left\{-\frac{1}{2}, \frac{1}{2}\right\}} z_{1}^{\alpha} z_{2}^{\beta}=\frac{1}{4} \prod_{\alpha=1}^{2}\left(z_{\alpha}^{\frac{1}{2}}+z_{\alpha}^{-\frac{1}{2}}\right)$, which generalises at higher orders, yielding an expression of the eigenvalues in terms of the families of parameters $\left(t_{\alpha}\right)_{1 \leq \alpha \leq n}$ only:

$$
\theta_{\mu}=\prod_{\alpha=1}^{n} \frac{\left(z_{\alpha}^{-\frac{1}{2}}+z_{\alpha}^{-\frac{1}{2}}\right)}{2}=\left(\prod_{\alpha=1}^{n} \frac{1}{1-t_{\alpha}^{2}}\right)^{\frac{1}{2}}
$$

Going back to the expression of the moment $\overline{Z_{t}^{n}}$ in Eq. 24, let us use again the fact that at time $t=0$ the allowed paths are just points, and are identical to the ones contributing to the partition function in the model with two fixed ends. If $\mu$ denotes a system of strings whose numbers of particles sum to $n$, the quantity $\left\langle\mu \mid \psi_{0}\right\rangle$ is therefore the combinatorial factor already calculated in 47 and reads

$$
\left\langle\mu \mid \psi_{0}\right\rangle=n !
$$


Imposing $L$-periodic boundary conditions induces the following equations for the family of rapidities:

$$
e^{i \lambda_{\alpha} L}=\prod_{\beta \neq \alpha} \frac{2 t_{\alpha}-2 t_{\beta}+\bar{c}}{2 t_{\beta}-2 t_{\alpha}-\bar{c}}, \quad 1 \leq \alpha \leq n
$$

which are related to the Bethe equations of the continuum Lieb-Liniger model by examining the role played by the parameters $t_{\alpha}$ in the Ansatz (Eq. 27).

Moreover, complex solutions to these equations in the thermodynamic limit (of a large number $L$ of sites), when expressed in terms of the tangents of the rapidities (and not in terms of the rapidities themselves as in the Lieb-Liniger model) are arranged in strings in the complex plane. Indeed, if the rapidity $\lambda_{\alpha}$ has a non-zero imaginary part, the l.h.s. of Eq. 32 goes to zero exponentially as a function of $L$, which implies that one of the factors in the r.h.s. must be zero in the thermodynamic limit, in the numerator (resp. in the denominator) if the imaginary part is positive (resp. negative). This implies that there exists an index $\beta$ such that $2 t_{\beta}=2 t_{\alpha} \pm \bar{c} / 2$ (with the optional sign identical to the sign of the imaginary part of $\lambda_{\alpha}$ ), up to corrections vanishing exponentially $\left.\right|^{3}$ at large $L$. Iterating this procedure yields a string with $m_{j}$ particles is specified by $m_{j}$ values corresponding to the family of parameters

$$
t_{\alpha}=t_{j, a}=i \frac{k_{j}}{2}+\frac{\bar{c}}{4}\left(m_{j}+1-2 a\right), \quad a \in\left\{1, \ldots, m_{j}\right\}
$$

where the quantity $k_{j}$ corresponds at leading order (in a continuum scaling limit involving small rapidities and large $\gamma$, and worked out in Section 5 of [47]) to the momentum of a string of rapidities in the Lieb-Liniger model. By an abuse of language, $k_{j}$ will sometimes be referred to as momentum in this paper (without taking the Lieb-Liniger limit).

\subsection{Expansion of the generating function in terms of strings}

At fixed number $n$ of variables, each of the Bethe eigensates needed to calculate the moment of order $n$ in the thermodynamic limit can be organised into a certain integer number of strings, denoted by $n_{s}$, and the numbers of particles contained in these strings must sum to $n$, so that with the above notations

$$
n=\sum_{j=1}^{n_{s}} m_{j}
$$

On the other hand, at fixed number $n_{s}$ of strings, one can find Bethe eigenstates with any integer number of variables. States with a fixed number of strings can therefore contribute to an infinity terms in formal the expansion of the generating function in terms of moments. Following the strategy of [14, 15, 47, 49], we reorganise the expansion as a sum over the number of strings:

$$
g_{\lambda}^{m o m}(u)=: 1+\sum_{n_{s} \geq 1} \frac{1}{n_{s} !} Z^{m o m}\left(n_{s}, u\right)=1+\sum_{n \geq 1} \frac{\overline{Z_{t}^{n}}}{n !}(-1)^{n} e^{-\lambda n u}
$$

whose consistency with the definition of $g_{\lambda}^{m o m}$ in Eq. 11 induces the definition of the quantity $Z^{m o m}\left(n_{s}, u\right)$, the contribution of the $n_{s}$ string sector to the generating function.

\footnotetext{
${ }^{3}$ All the results of this paper are derived in the thermodynamic limit, neglecting corrections to the rapidities depending on the size of the sysem.
} 


\section{Localisation on parity-invariant string states}

\subsection{Symmetry argument from the overlap factor}

In [31], it was argued that only parity-invariant systems of strings (systems of strings of rapidities in which no rapidity can be found without its opposite to match) have non-zero overlap with the uniform wave function in the Lieb-Liniger model in the thermodynamic limit. This symmetry argument provided a selection rule that confirmed the results of the detailed calculations of [15], in which the partition sums happened to localise on such systems of strings. As the mapping from rapidities to the $t_{\alpha}$ parameters (Eq. 28) is odd, the same localisation should hold in the thermodynamic limit of the log-gamma polymer.

In the simplest possible case of one string with two particles, we can check this explicity by parametrizing the system with one real parameter $k$ :

$$
\begin{gathered}
t_{1}=i k+\frac{\bar{c}}{4}, \quad t_{2}=i k-\frac{\bar{c}}{4}, \\
z_{1}=e^{i \lambda_{1}}=\frac{4+\bar{c}+i k}{4-\bar{c}-i k}=\left(z_{2}^{*}\right)^{-1}=: \rho e^{i \theta},
\end{gathered}
$$

with $\rho>1$ and $\theta$ in the interval $[0,2 \pi[$. The overlap of this string state reads

$$
\sum_{x_{1}, x_{2} \in\{0, \ldots, L-1\}} \Psi_{k, m=2}=2\left(2 \sum_{x_{1}=0}^{L-1} \sum_{x_{2}=x_{1}}^{L}\left(\rho^{-1}\right)^{x_{2}-x_{1}}\left(e^{i \theta}\right)^{x_{1}+x_{2}}+\sum_{x_{1}=0}^{L-1} e^{2 i \theta x_{1}}\right) .
$$

Geometric sums allow to express at finite $L$ (in the case $\theta \neq 0$ ) ratios of the form

$$
\frac{1}{L} \sum_{x=0}^{L} e^{i x \theta}=\frac{1}{L} \frac{1-e^{i(L+1) x \theta}}{1-e^{i x \theta}}=\frac{e^{i \frac{L x \theta}{2}}}{L} \frac{\sin ((L+1) x \theta / 2)}{\sin (x \theta / 2)},
$$

which goes to zero when $L$ goes to infinity. On the other hand, if $\theta=0$ the l.h.s of the above equation is identically equal to 1 , hence

$$
\lim _{L \rightarrow \infty}\left(\frac{1}{L} \sum_{x=0}^{L} e^{i x \theta}\right)=\delta_{e^{i \theta}, 1} .
$$

This identity implies that the contribution of the string goes to zero in the thermodynamic limit if $z_{1}$ is not real, which condition is equivalent to $k=0$. See Section 4 for the calculation of the overlap in the case of real parameters $t_{1}$ and $t_{2}$ (corresponding to $k=0$ ), that contributes to the $\overline{Z_{t}^{2}}$.

We have to express the contribution to the generating function of systems of perfect strings consisting of $M$ strings with real parameters $t_{\alpha}$, described using $M$ integers $m_{1}, \ldots, m_{M}$ corresponding to the number of particles in each of these strings, and $N$ pairs of strings, described by $N$ real parameters $k_{1}, \ldots, k_{N}$ (paired with their opposites $-k_{1}, \ldots,-k_{N}$ ) and $N$ integers $n_{1}, \ldots, n_{N}$ corresponding to the number of particles in each of the elements of each of the $N$ pairs of strings. This structure is identical to the structure of the strings of rapidities on which the overlaps localise in the Lieb-Liniger model [31]. 


\subsection{Time-evolution factors of parity-invariant string states}

For an eigenstate consisting of one string, the eigenvalue of the time-evolution operator can be obtained by substituting the string structure described in Eq. 33 into the expression of eigenvalues in Eq. 30. This substition yields

$$
\theta_{m, k}=\left(\prod_{a=1}^{m} \frac{1}{1-t_{a}^{2}}\right)^{\frac{1}{2}}=\left(\frac{2}{\bar{c}}\right)^{m}\left(\frac{\Gamma\left(-\frac{m}{2}+\frac{\gamma}{2}-i \frac{k}{\bar{c}}\right) \Gamma\left(-\frac{m}{2}+\frac{\gamma}{2}+i \frac{k}{\bar{c}}\right)}{\Gamma\left(\frac{m}{2}+\frac{\gamma}{2}-i \frac{k}{\bar{c}}\right) \Gamma\left(\frac{m}{2}+\frac{\gamma}{2}+i \frac{k_{j}}{\bar{c}}\right)}\right)^{\frac{1}{2}},
$$

where the identity $\prod_{k=0}^{m-1}(a+k)=\Gamma(a+m) / \Gamma(a)$ has been used, together with the relation $\gamma=1+4 / \bar{c}$ that follows from the definition of the charge $\bar{c}$ in Eq. 17.

In the parity-invariant system of strings described above, the time-evolution factor is obtained from products of the above expression:

$$
\theta_{\mu \equiv\left\{m_{j}, n_{p}, k_{p}\right\}_{1 \leq j \leq M, 1 \leq p \leq N}}=\prod_{p=1}^{N} \theta_{n_{p}, k_{p}} \theta_{n_{p},-k_{p}} \prod_{j=1}^{M} \theta_{m_{j}, 0}
$$

Using the expression 41 in terms of Gamma functions, we obtain:

$\theta_{\mu \equiv\left\{m_{j}, n_{p}, k_{p}\right\}}=\left(\frac{2}{\bar{c}}\right)^{2 \sum_{p=1}^{N} n_{p}+\sum_{j=1}^{M} m_{j}} \prod_{p=1}^{N}\left(\frac{\Gamma\left(-\frac{n_{p}}{2}+\frac{\gamma}{2}-i \frac{k_{p}}{\bar{c}}\right) \Gamma\left(-\frac{n_{p}}{2}+\frac{\gamma}{2}+i \frac{k_{p}}{\bar{c}}\right)}{\Gamma\left(\frac{n_{p}}{2}+\frac{\gamma}{2}-i \frac{k_{p}}{\bar{c}}\right) \Gamma\left(\frac{n_{p}}{2}+\frac{\gamma}{2}+i \frac{k_{p}}{\bar{c}}\right)}\right)^{\frac{1}{2}} \prod_{j=1}^{M}\left(\frac{\Gamma\left(-\frac{n_{p}}{2}+\frac{\gamma}{2}\right)}{\Gamma\left(\frac{n_{p}}{2}+\frac{\gamma}{2}\right)}\right)$.

\subsection{Norm of the parity-invariant states and overlap with the uniform wave function}

\subsubsection{Norms of the string states of the log-gamma model}

The formula for the norm of the systems of $n_{s}$ strings in the Lieb-Liniger model, conjectured by Gaudin [6] and proved by Korepin [50] was generalised in Section 7.5 of [47] into a form that reduces to the Gaudin formula in the Lieb-Liniger limit. The squared norm of a state $|\mu\rangle$ consisting of a system of $n_{s}$ perfect strings with momenta $k_{1}, \ldots, k_{n_{s}}$ and numbers of particles $m_{1}, \ldots, m_{n_{s}}$, for a total number of particles denoted by $n=\sum_{i=1}^{n_{s}} m_{i}$, reads as the product of an inter-string factor and an intra-string factor

$$
\langle\mu \mid \mu\rangle=n ! L^{n_{s}} \prod_{1 \leq i<j \leq n_{s}} \frac{4\left(k_{i}-k_{j}\right)^{2}+\bar{c}^{2}\left(m_{i}+m_{j}\right)^{2}}{4\left(k_{i}-k_{j}\right)^{2}+\bar{c}^{2}\left(m_{i}-m_{j}\right)^{2}} \prod_{j=1}^{n_{s}}\left(\frac{m_{j}}{\bar{c}^{m_{j}-1}}\left(\sum_{a=1}^{m_{j}} \frac{1}{1-t_{j, a}^{2}}\right) \prod_{b=1}^{m_{j}}\left(1-t_{j, b}^{2}\right)\right),
$$

which reproduces the Lieb-Liniger result by formally by setting all the parameters $t_{\alpha}$ to zero. Let us call the second product over strings in the above formula the deformation factor, and introduce the notation

$$
\tau_{m_{j}, k_{j}}=\sum_{a=1}^{m_{j}} \frac{1}{1-t_{j, a}^{2}},
$$


where the mapping from the pair $\left(m_{j}, k_{j}\right)$ to the set of $m_{j}$ numbers $\left(t_{j, a}\right)_{1 \leq a \leq m_{j}}$, is as always given by the definition of strings in the thermodynamic limit (Eq. 33). Using the notation introduced in Eq. 41 for the eigenvalue associated to a single string yields

$$
\langle\mu \mid \mu\rangle=\left(\left(\sum_{j=1}^{n_{s}} m_{j}\right) !\right) L^{n_{s}} \prod_{1 \leq i<j \leq n_{s}} \frac{4\left(k_{i}-k_{j}\right)^{2}+\bar{c}^{2}\left(m_{i}+m_{j}\right)^{2}}{4\left(k_{i}-k_{j}\right)^{2}+\bar{c}^{2}\left(m_{i}-m_{j}\right)^{2}} \prod_{j=1}^{n_{s}}\left(\frac{m_{j}}{\bar{c}^{m_{j}-1}} \tau_{m_{j}, k_{j}} \theta_{m_{j}, k_{j}}^{-2}\right) .
$$

Specialising this expression to a system of $M$ strings with zero momentum yields

$$
\left\|\mu \equiv\left\{k=0, m_{1}, \ldots, m_{M}\right\}\right\|^{2}=\left(\left(\sum_{i=1}^{M} m_{i}\right) !\right) L^{M} \prod_{1 \leq i<j \leq M} \frac{\left(m_{i}+m_{j}\right)^{2}}{\left(m_{i}-m_{j}\right)^{2}} \prod_{j=1}^{M}\left(\frac{m_{j}}{\bar{c}^{m_{j}-1}} \tau_{m_{j}, 0} \theta_{m_{j}, 0}^{-2}\right) .
$$

On the other hand, for the above-described parity-invariant system of $N$ pairs of strings, the intra-string factor depending on the $2 \sum_{j=1}^{N} m_{j}$ parameters $t_{\alpha}$ depends only on the squares of these parameters, which take half as many distinct values, which is reflected in our notations for each pair of strings by the fact that the quantities $\theta$ and $\tau$ entering the deformation factor are even functions of the momentum:

$$
\theta_{m_{j}, k_{j}}=\theta_{m_{j},-k_{j}}, \quad \tau_{m_{j}, k_{j}}=\tau_{m_{j},-k_{j}}, \quad j=1, \ldots, N
$$

The deformation factor can therefore be expressed in terms of the factors that would arise by specialising Eq. 44 to a system of $N$ strings that give rise to all the distinct values of the parameters $t_{\alpha}$, say the strings that have the momenta $k_{1}, \ldots k_{N}$ :

$$
\begin{aligned}
\| \mu \equiv & \left\{\left\{k_{1},-k_{1}, \ldots, k_{N},-k_{N}\right\},\left\{n_{1}, n_{1}, \ldots, n_{N}, n_{N}\right\}\right\} \|^{2}=\left(\left(2 \sum_{p=1}^{N} n_{p}\right) !\right) L^{2 N} \\
& \times \prod_{1 \leq p<q<N}\left(\frac{4\left(k_{p}-k_{q}\right)^{2}+\left(n_{p}+n_{q}\right) \bar{c}^{2}}{4\left(k_{p}-k_{q}\right)^{2}+\left(n_{p}-n_{q}\right) \bar{c}^{2}} \frac{4\left(k_{p}+k_{q}\right)^{2}+\left(n_{p}+n_{q}\right) \bar{c}^{2}}{4\left(k_{p}+k_{q}\right)^{2}+\left(n_{p}-n_{q}\right) \bar{c}^{2}}\right) \prod_{p=1}^{N} \frac{4 k_{p}^{2}+n_{p}^{2} \bar{c}^{2}}{4 k_{p}^{2}} \\
& \times \prod_{p=1}^{N}\left(\left(\frac{n_{p}}{\bar{c}^{n_{p}-1}}\right)^{2} \tau_{n_{p}, k_{p}}^{2} \theta_{n_{p}, k_{p}}^{-4}\right) .
\end{aligned}
$$

The squared norm of the most general relevant set of strings $|\mu\rangle$ can therefore be written, up to a combinatorial factor involving the total number of particles, as the product of the two above squared norms by the missing inter-string factors:

$$
\begin{aligned}
\langle\mu \mid \mu\rangle & =\left(\left(2 \sum_{p=1}^{N} n_{p}+\sum_{j=1}^{M} m_{j}\right) !\right) L^{2 N+M} \prod_{1 \leq i<j \leq M} \frac{\left(m_{i}+m_{j}\right)^{2}}{\left(m_{i}-m_{j}\right)^{2}} \prod_{p=1}^{N} \frac{4 k_{p}^{2}+n_{p}^{2} \bar{c}^{2}}{4 k_{p}^{2}} \\
& \times \prod_{1 \leq p<q<N}\left(\frac{4\left(k_{p}-k_{q}\right)^{2}+\left(n_{p}+n_{q}\right) \bar{c}^{2}}{4\left(k_{p}-k_{q}\right)^{2}+\left(n_{p}-n_{q}\right) \bar{c}^{2}} \frac{4\left(k_{p}+k_{q}\right)^{2}+\left(n_{p}+n_{q}\right) \bar{c}^{2}}{4\left(k_{p}+k_{q}\right)^{2}+\left(n_{p}-n_{q}\right) \bar{c}^{2}}\right) \\
& \times \prod_{1 \leq p<N, 1 \leq j \leq M} \frac{4 k_{p}^{2}+\left(n_{p}+m_{j}\right) \bar{c}^{2}}{4 k_{p}^{2}+\left(n_{p}-m_{j}\right) \bar{c}^{2}} \\
& \times \prod_{j=1}^{M}\left(\frac{m_{j}}{\bar{c}^{m_{j}-1}} \tau_{m_{j}, 0} \theta_{m_{j}, 0}^{-2}\right) \prod_{p=1}^{N}\left(\left(\frac{n_{p}}{\bar{c}^{n_{p}-1}}\right)^{2} \tau_{n_{p}, k_{p}}^{2} \theta_{n_{p}, k_{p}}^{-4}\right)
\end{aligned}
$$




\subsubsection{Form of the overlaps}

In the case of a parity-invariant system of strings in the Lieb-Liniger model, consisting of a pair of strings with opposite momenta, it was argued in [31, 49], that the overlap with the uniform wave function in the Lieb-Liniger model depends on the squared norm of just one string of particles (all with the same momentum), and each parity invariant single strings with zero momentum was related to the zero-momentum limit of such a pair of strings. We conjecture that the same relation holds in the log-gamma model, up to deformations ensuring the factors of $\tau$ compensate the contribution of measure-theoretic factors after integration over phase space (see Subsection 3.4), so that the squared norms of the string states in the log-gamma model (Eq. 49) induce the overlap

$$
\begin{aligned}
\Omega_{\mu} & =\frac{n !(L \bar{c})^{M+N}}{\bar{c}^{n}} \frac{2^{n}}{2^{M}}\left(\prod_{j=1}^{M} \frac{m_{j}}{m_{j} !} \tau_{m_{j}, 0} \theta_{m_{j}, 0}^{-1}\right) \prod_{1 \leq i<j \leq M}(-1)^{\min \left(m_{i}, m_{j}\right)} \operatorname{sgn}\left(m_{i}-m_{j}\right) \frac{m_{i}+m_{j}}{\left|m_{i}-m_{j}\right|} \\
& \left(\times \prod_{p=1}^{N}(-1)^{n_{p}} n_{p} \tau_{n_{p}, k_{p}} \theta_{n_{p}, k_{p}}^{-2} \prod_{q=0}^{n_{p}-1} \frac{1}{4 k_{p}^{2} / \bar{c}^{2}+q^{2}}\right) \prod_{1 \leq p<N, 1 \leq j \leq M} \frac{4 k_{p}^{2}+\left(n_{p}+m_{j}\right) \bar{c}^{2}}{4 k_{p}^{2}+\left(n_{p}-m_{j}\right) \bar{c}^{2}} \\
& \times \prod_{1 \leq p<q<N}\left(\frac{\left.4\left(k_{p}-k_{q}\right)^{2}+\left(n_{p}+n_{q}\right) \bar{c}^{2}\right)}{\left.4\left(k_{p}-k_{q}\right)^{2}+\left(n_{p}-n_{q}\right) \bar{c}^{2}\right)} \frac{4\left(k_{p}+k_{q}\right)^{2}+\left(n_{p}+n_{q}\right) \bar{c}^{2}}{4\left(k_{p}+k_{q}\right)^{2}+\left(n_{p}-n_{q}\right) \bar{c}^{2}}\right) .
\end{aligned}
$$

As a check, consider the simplest case needed for computation of the one-string contribution, namely that of one string with $m=2$ particles:

$$
\begin{gathered}
t_{1}=\frac{\bar{c}}{4}, \quad t_{2}=-\frac{\bar{c}}{4}, \\
z_{1}=e^{i \lambda_{1}}=\frac{4+\bar{c}}{4-\bar{c}}=\frac{\gamma}{\gamma-2}=e^{-i \lambda_{2}}=z_{2}^{-1} .
\end{gathered}
$$

The combinatorial factors, corresponding to the permutations of two objects denoted by (12) and (21), read

$$
A_{(12)}=1+\operatorname{sign}\left(x_{2}-x_{1}+0^{+}\right)=2 \delta_{x_{2}>x_{1}}+2 \delta_{x_{2}=x_{1}},
$$

and

$$
A_{(21)}=1-\operatorname{sign}\left(x_{2}-x_{1}+0^{+}\right)=2 \delta_{x_{2}<x_{1}},
$$

so that

$$
\begin{aligned}
\Psi_{m=2}\left(x_{1}, x_{2}\right) & =2 e^{i\left(\lambda_{1} x_{1}+\lambda_{2} x_{2}\right)} \delta_{x_{2}>x_{1}}+2 \delta_{x_{2}=x_{1}}+2 e^{i\left(\lambda_{1} x_{2}+\lambda_{2} x_{1}\right)} \delta_{x_{2}<x_{1}} \\
& =2 z_{1}^{x_{1}-x_{2}} \delta_{x_{2}-x_{1}>0}+2 \delta_{x_{2}=x_{1}}+2 z_{1}^{x_{2}-x_{1}} \delta_{x_{2}-x_{1}<0}
\end{aligned}
$$


and the overlap reads, introducing the variable $y=x_{2}-x_{1}$ to perform the sums on the lattice:

$$
\begin{aligned}
\sum_{x_{1}=0}^{L-1} \sum_{x_{2}=0}^{L-1} \Psi_{m=2}\left(x_{1}, x_{2}\right) & =\sum_{x_{1}=0}^{L-1} \Psi_{m=2}\left(x_{1}, x_{1}\right)+\sum_{x_{1}=0}^{L-1} \sum_{-x_{1} \leq y \leq L-1-x_{1}, y \neq 0} \Psi_{m=2}\left(x_{1}, y+x_{1}\right) \\
& =2 L+2 \sum_{x_{1}=0}^{L-2} \sum_{1 \leq y \leq L-1-x_{1}} z_{1}^{-y}+2 \sum_{x_{1}=1}^{L-1} \sum_{-x_{1} \leq y \leq-1} z_{1}^{y} \\
& =2 L+2 z_{1}^{-1} \sum_{x_{1}=0}^{L-2} \frac{1-z_{1}^{-\left(L-1-x_{1}\right)}}{1-z_{1}^{-1}}+2 z_{1}^{-1} \sum_{x_{1}=1}^{L-1} \frac{1-z_{1}^{-x_{1}}}{1-z_{1}^{-1}} \\
& =2 L+4(L-1) \frac{z_{1}^{-1}}{1-z_{1}^{-1}}-2 \frac{z_{1}^{-L}}{1-z_{1}^{-1}} \sum_{x_{1}=0}^{L-2} z_{1}^{x_{1}}-2 \frac{z_{1}^{-1}}{1-z_{1}^{-1}} \sum_{x_{1}=1}^{L-1} z_{1}^{-x_{1}} \\
& =2 L+4(L-1) \frac{z_{1}^{-1}}{1-z_{1}^{-1}}-2 \frac{z_{1}^{-L}}{1-z_{1}^{-1}} \frac{1-z_{1}^{L-1}}{1-z_{1}}-2 \frac{z_{1}^{-1}}{1-z_{1}^{-1}} \frac{z_{1}^{-1}\left(1-z_{1}^{-(L-1)}\right)}{1-z_{1}^{-1}}
\end{aligned}
$$

The dominant term in this expression is proportional to $L$, and as $z_{1}>1$ the partial sums of geometric series converge, so that at large $L$ we obtain

$$
\begin{aligned}
\sum_{x_{1}=0}^{L-1} \sum_{x_{2}=0}^{L-1} \Psi_{m=2}\left(x_{1}, x_{2}\right) & =2(\gamma-1) L-(\gamma-2)\left(\frac{3 \gamma}{2}-1\right)+z_{1}^{-L} \\
& =\frac{8 L}{\bar{c}}\left(1+\frac{1}{8 \bar{c} L}(\bar{c}-4)(\bar{c}+6)+O\left(L^{-1} z_{1}^{-L}\right)\right) .
\end{aligned}
$$

On the other hand, specialising Eq. 51 to the case $M=1, m_{1}=2, N=0$ yields a total number of particles $n=2$, and the conjectured expression for the thermodynamic limit of the overlap

$$
\Phi_{m=2, k=0}=\frac{4(L \bar{c})}{\bar{c}^{2}} \tau_{2,0} \theta_{2,0}^{-1}
$$

which coincides with the large- $L$ limit of the overlap (Eq. 58), as

$$
\tau_{2,0}=\frac{2}{1-t_{1}^{2}} \quad \text { and } \quad \theta_{2,0}^{-1}=\left(\frac{1}{\left(1-t_{1}^{2}\right)^{2}}\right)^{-\frac{1}{2}} \text {. }
$$

\subsection{Measure-theoretic factors}

The sum over Bethe eigenstates in Eq. 24 involves an integration over the space of rapidities. The expression of the moment $\overline{Z_{t}^{n}}$ as integral over the parameters $\left(k_{j}\right)_{1 \leq j \leq n_{s}}$ therefore contains a Jacobian factor induced by the string structure of the eigenstates, and Eq. 28 relating the string states to rapidities. This factor has been worked out in [47] in the case of the log-gamma polymer with fixed end, which does not involve the constraint of parity invariance on string states. In the case of free ends, the constraints are implemented by $\delta$-measure factors of two kinds, with arguments containing either a single linear momentum or a pair of linear momenta, and appeared in [15. We therefore have to work out the Jacobian factors in the log-gamma case, in which the parameters $k$ of strings are not identical to sums of rapidities. 


\subsubsection{Pairs of strings with identical number of particles and opposite linear momenta}

Consider a parity-invariant pair of strings. They have the same number $m$ of particles and can be denoted as follows:

$$
\begin{aligned}
& t_{1, a}=i \frac{k_{1}}{2}+\frac{\bar{c}}{4}(m+1-2 a), \quad a \in\{1, \ldots, m\}, \\
& t_{2, a}=i \frac{k_{2}}{2}+\frac{\bar{c}}{4}(m+1-2 a), \quad a \in\{1, \ldots, m\}, \quad k_{2}=-k_{1} .
\end{aligned}
$$

To sum over all such Bethe eigenstates, we have to integrate over the corresponding rapidities, which are also parity-invariant combinations, with the same number of elements, giving rise to the measure in phase space

$$
\sum_{\mu, n_{s}=2, m_{1}=m_{2}=m, k_{1}+k_{2}=0} \longrightarrow \prod_{a=1}^{m} d \lambda_{1, a} d \lambda_{2, a} \delta\left(\sum_{a=1}^{m}\left(\lambda_{1, a}+\lambda_{2, a}\right)\right),
$$

so that the constraint ensures $k_{1}=-k_{2}$ in the string space. Changing variables to $k_{1}$ and $k_{2}$ gives rise to Jacobian factors:

$$
\varphi\left(k_{1}, k_{2}\right):=\sum_{a=1}^{m}\left(\lambda_{1, a}+\lambda_{2, a}\right)=\frac{1}{i} \log \left(\prod_{a=1}^{m} \frac{\left(4+2 i k_{1}+\bar{c}(m+1-2 a)\right)\left(4+2 i k_{2}+\bar{c}(m+1-2 a)\right)}{\left(4-2 i k_{1}+\bar{c}(m+1-2 a)\right)\left(4-2 i k_{2}+\bar{c}(m+1-2 a)\right)}\right) .
$$

In sums over parity-invariant states containing several parity-invariant pairs of strings, each of the strings contributes such a measure-theoretic factor:

$$
\left.\frac{\partial}{\partial k_{2}} \varphi\left(k_{1}, k_{2}\right)\right|_{k_{2}=-k_{1}}=\sum_{a=1}^{m} \frac{1}{1-t_{1, a}^{2}}=\tau_{m, k_{1}},
$$

where we used the notation introced in Eq. 45. The localisation constraint in the rapidity space can therefore be expressed in terms of a constraint in the space of momenta with coordinates $k_{1}$ ans $k_{2}$ as

$$
\delta\left(\sum_{a=1}^{m}\left(\lambda_{1, a}+\lambda_{2, a}\right)\right)=\frac{1}{\tau_{m, k_{1}}} \delta\left(k_{1}+k_{2}\right) .
$$

On the other hand, the sum over string states transforms as:

$$
\prod_{a=1}^{m} d \lambda_{i, a}=\left(\sum_{a=1}^{m} \frac{1}{1-t_{i, a}^{2}}\right) d k_{i}=\tau_{m, k_{i}} d k_{i}
$$

which is the transformation of the Lebesgue measure on phase space that was worked out in Section 7 of [47]. But the parity-invariance constraint $k_{1}=-k_{2}$ implies that $\tau_{m, k_{1}}=\tau_{m, k_{2}}$. There is therefore a net measure-theoretic factor when integrating over the parameters $k_{1}$ and $k_{2}$ with the parity-invariance constraint:

$$
\prod_{a=1}^{m} d \lambda_{1, a} d \lambda_{2, a} \delta\left(\sum_{a=1}^{m}\left(\lambda_{1, a}+\lambda_{2, a}\right)\right)=d k_{1} d k_{2} \delta\left(k_{1}+k_{2}\right) \sum_{a=1}^{m} \frac{1}{1-t_{1, a}^{2}} .
$$




\subsubsection{One string with zero momentum}

Let us call $m$ the number of particles in a string solution to the Bethe equations, with zero parameter $k$ (and drop the index $j$ labeling the string), in the thermodynamic limit, at leading order in the size of the system (dropping the exponentially small corrections):

$$
t_{a}=i \frac{k}{2}+\frac{\bar{c}}{4}(m+1-2 a), \quad \text { with } \quad k=0, \quad a \in\{1, \ldots, m\} .
$$

As the mapping from the rapidities to these parameters is odd, summing over all such string states involves integrating against the phase space measure of all the corresponding rapidities:

$$
\lambda_{a}=\frac{1}{i} \log \left(\frac{4+2 i k+\bar{c}(m+1-2 a)}{4-2 i k+\bar{c}(m+1-2 a)}\right) .
$$

The sum of rapidities of all the particles in a string

$$
\sum_{a=1}^{m} \lambda_{a}=\frac{1}{i} \log \prod_{a=1}^{m}\left(\frac{4+2 i k+\bar{c}(m+1-2 a)}{4-2 i k+\bar{c}(m+1-2 a)}\right) .
$$

is zero if and only if $k=0$, since the family of integers $\{m+1-2 a\}_{1 \leq a \leq m}$ is parity-invariant. The measure-theoretic factor therefore yields a delta-function of the scalar parameter $k$, up to a division by:

$$
\left.\frac{\partial}{\partial k} \prod_{a=1}^{m}\left(\frac{4+2 i k+\bar{c}(m+1-2 a)}{4-2 i k+\bar{c}(m+1-2 a)}\right)\right|_{k=0}=4 i \sum_{a=1}^{m} \frac{1}{4+\bar{c}(m+1-2 a)}=\frac{i}{1+t_{a}}
$$

so that the integration measure over the phase space of the one-string parity-invariant sector reads (using again Eq. 66 for the Lebesgue measure):

$$
\prod_{a=1}^{m} d \lambda_{a} \delta\left(\sum_{a=1}^{m} \lambda_{a}\right)=\tau_{m, 0} d k \frac{1}{\left|\sum_{a=1}^{m} \frac{1}{1+t_{a}}\right|} \delta(k)
$$

The above expression can be related to the quantity $\tau_{m, 0}$ using the parity invariance of the family of $t_{a}$ parameters:

$$
\sum_{a=1}^{m} \frac{1}{1+t_{a}}=\frac{1}{2} \sum_{a=1}^{m}\left(\frac{1}{1+t_{a}}+\frac{1}{1-t_{a}}\right)=\frac{1}{2} \sum_{a=1}^{m} \frac{2}{1-t_{a}^{2}}=\tau_{m, 0},
$$

yielding the measure-theoretic contribution

$$
\prod_{a=1}^{m} d \lambda_{a} \delta\left(\sum_{a=1}^{m} \lambda_{a}\right)=\frac{\tau_{m, 0}}{\left|\tau_{m, 0}\right|} \delta(k) d k .
$$

The constraint $\delta(k)$ ensures that the string has zero imaginary part. In sums over parity-invariant states containing several strings with zero momentum, each of the strings contributes such a measure-theoretic factor. These factors compensate the factors from the Lebesgue measure, up to a sign, which can be fixed by requiring that setting all the $t_{\alpha}$ parameters to 0 should yield the Lieb-Liniger formula. 


\section{The one-string contribution to the generating function at large time}

Using the measure worked out in Eq. 74, leaves the sum over eigenstates initially labeled by $\mu$ as a sum over the number of particles $m$ in the string states (as in does in the continuum Lieb-Liniger model with one free-end in [15], up to deformation factors coming from the squared norms of the string states and from the overlap factors). From the definition of the string expansion of the generating function in terms of integer moments in Eq. 35, we find the formal series

$$
Z^{m o m}\left(n_{s}=1, u\right)=\sum_{m=1}^{\infty} \frac{(-1)^{m} e^{-\lambda m u}}{m !}\left(2^{m t}\left(\frac{\bar{c}}{4}\right)^{m(t+1)} \theta_{m, 0}^{t} \frac{m !}{\langle k=0, m \mid k=0, m\rangle} \Omega_{m, 0}\right)
$$

where use has been made of the first term of the string expansion of each moment $\overline{Z_{t}^{m}}$, read off from Eq. 24, and specialised to a string of $m$ particles with $k=0$, together with the combinatorial factor of Eq. 31. Moreover, the square of the norm of the single-string state for all values of the momentum and of the number of particles is obtained as a particular case of Eq. 44 ;

$$
\langle k=0, m \mid k=0, m\rangle=m ! \frac{m L}{\bar{c}^{m-1}} \tau_{m, 0} \theta_{m, 0}^{-2},
$$

and the overlap is read off by specialising Eq. 51 to the case of one string with $m$ particles

$$
\Omega_{m, 0} \sim_{L \rightarrow \infty} \frac{2^{m-1} m L}{\bar{c}^{m-1}} \tau_{m, 0} \theta_{m, 0}^{-1}
$$

Moreover the time-dependent contribution can be read off from Eq. 43

$$
\theta_{k=0, m}=\left(\frac{2}{\bar{c}}\right)^{m}\left(\frac{\Gamma\left(-\frac{m}{2}+\frac{\gamma}{2}\right)}{\Gamma\left(\frac{m}{2}+\frac{\gamma}{2}\right)}\right) .
$$

The factors of $\sum_{a=1}^{m_{j}} \frac{1}{1-t_{j, a}^{2}}$ come in inverse powers from the Lebesgue measure and delta-function on one side, and from the overlap and squared norm of the string state on the other side. The onestring contribution is therefore given by a sum over the number of particles:

$$
Z^{m o m}\left(n_{s}=1, u\right)=\frac{1}{2} \sum_{m=1}^{\infty}(-1)^{m} 2^{m(t+1)}\left(\frac{\bar{c}}{4}\right)^{m(t+1)} \frac{1}{m !}\left(\frac{2}{\bar{c}}\right)^{m(t+1)}\left(\frac{\Gamma\left(-\frac{m}{2}+\frac{\gamma}{2}\right)}{\Gamma\left(\frac{m}{2}+\frac{\gamma}{2}\right)}\right)^{t+1} e^{-\lambda m u} .
$$

The analytic continuation to complex values of $m$ inspired by the Mellin representation of the exponential function [47] yields the integral form of the one-string contribution to the denerating function:

$$
Z\left(n_{s}=1, u\right)=-\frac{1}{2} \int_{C} \frac{d s}{2 i \sin (\pi s)} e^{-\lambda u s} \frac{1}{\Gamma(s+1)}\left(\frac{\Gamma\left(-\frac{s}{2}+\frac{\gamma}{2}\right)}{\Gamma\left(\frac{s}{2}+\frac{\gamma}{2}\right)}\right)^{t+1},
$$

where $C=a+i \mathbf{R}$, in the notations of Eq. 13. Let us define the function

$$
J_{\gamma}(s):=\log \left(\frac{\Gamma\left(-\frac{s}{2}+\frac{\gamma}{2}\right)}{\Gamma\left(\frac{s}{2}+\frac{\gamma}{2}\right)}\right) .
$$


Rescaling the integration variable by a factor of $\lambda$ by the change of variable $\tilde{s}=\lambda s$ should allow us use the saddle-point method in the large- $\lambda$ limit. The one-string contribution to the generating function therefore reads

$$
Z\left(n_{s}=1, u\right)=-\frac{1}{2} \int_{C} \frac{d \tilde{s}}{2 i \lambda \sin \left(\pi \frac{\tilde{s}}{\lambda}\right)} \frac{1}{\Gamma\left(\frac{\tilde{s}}{\lambda}+1\right)} \exp \left(-\tilde{s} u+t^{\prime} J_{\gamma}\left(\frac{\tilde{s}}{\lambda}\right)\right),
$$

where $t^{\prime}=t+1$, which in the large-time limit is equivalent to time. The function $J_{\gamma}$ can be Taylor-expanded around $s=0$ as

$$
J_{\gamma}(s)=-\psi\left(\frac{\gamma}{2}\right) s-\frac{1}{24} \psi^{\prime \prime}\left(\frac{\gamma}{2}\right) s^{3}+O\left(s^{5}\right)
$$

where $\psi=\Gamma^{\prime} / \Gamma$ denotes the digamma function.

$$
Z\left(n_{s}=1, u\right)=-\frac{1}{2} \int_{C} \frac{d \tilde{s}}{2 i \lambda \sin \left(\pi \frac{\tilde{s}}{\lambda}\right)} \frac{1}{\Gamma\left(\frac{\tilde{s}}{\lambda}+1\right)} \exp \left(-\tilde{s} u-\psi\left(\frac{\gamma}{2}\right) \frac{\tilde{s}}{\lambda} t^{\prime}-\frac{t^{\prime}}{24 \lambda^{3}} \psi^{\prime \prime}\left(\frac{\gamma}{2}\right) \tilde{s}^{3}+O\left(t^{\prime} \lambda^{-5}\right)\right)
$$

Let us shift the energy by so that the term proportional to $t s$ in the argument of the exponential disappears, meaning that the large- $\lambda$ limit will address the distribution function of the random variable $\log Z_{t}+\psi(\gamma / 2) t$, whose random character is entirely due to $Z_{t}$. Let us adjust the parameter $\lambda$ using the Airy representation of the exponential of a cubic function (which in the continuum model was used to tame the rapid growth of the moments, that were defined at all orders but whose energy grows as the cube of the order, [15], whereas in the log-gamma model with fixed ends it was ). The identity

$$
e^{\frac{w^{3}}{3}}=\int_{-\infty}^{\infty} d y \operatorname{Ai}(y) e^{y w}
$$

should allow us to rewrite the cubic term in $\tilde{s}$ as an exponent that is linear in the parameter $\lambda$. With the choice

$$
\lambda^{3}=-\frac{1}{8} \psi^{\prime \prime}\left(\frac{\gamma}{2}\right) t
$$

the large-time and large- $\lambda$ limits become equivalent (and one can check that replacing $t^{\prime}$ with $t$ altogether in Eq. 84, with time scaling as the cube of $\lambda$, yields a correction of order $O\left(\lambda^{-3}\right.$, which is subdominant as $t \lambda^{-5} \sim_{\lambda \rightarrow \infty} \lambda^{-2}$ ). Going back to the definition of the rescaled free energy denoted by $f$ (in Eq. 8), and using the notation $f$ again for the rescaled free energy of the free-end model with the above-described shift in energy, we can write

$$
f=\frac{-2}{\left(\psi^{\prime \prime}\left(\frac{\gamma}{2}\right) t\right)^{\frac{1}{3}}}\left(\log Z_{t}+\psi\left(\frac{\gamma}{2}\right) t\right)
$$

The large- $\lambda$ limit of the one-string contribution $Z(1, u)$ of Eq. 84 , together with the definition of $\lambda$ as a function of time in Eq. 86, characterises the one-string contribution to the cumulative distribution function of the rescaled free energy $f$. The shift in the energy levels and the definition of $\lambda$ in terms of time and the parameter $\gamma$ we have just made induce all the higher-order contributions 
to the string expansion of the generating function.

Using the Airy representation of Eq. 85, we obtain the one-string contribution as a double integral, in which we can shift the argument of the Airy function by a change of variable:

$$
\begin{aligned}
& Z\left(n_{s}=1, u\right) \underset{\lambda \rightarrow \infty}{\sim} \frac{1}{2} \int_{-\infty}^{+\infty} d y \operatorname{Ai}(y) \int_{-a+i \mathbf{R}} \frac{d \tilde{s}}{2 i \lambda \sin \left(\pi \frac{\tilde{s}}{\lambda}\right)} \frac{1}{\Gamma\left(\frac{\tilde{s}}{\lambda}+1\right)} \exp \left((y-u) \tilde{s}+O\left(\lambda^{-2}\right)\right) \\
& \underset{\lambda \rightarrow \infty}{\sim} \frac{1}{2} \int_{-\infty}^{+\infty} d y \operatorname{Ai}(y+u) \int_{-a+i \mathbf{R}} \frac{d \tilde{s}}{2 i \lambda \sin \left(\pi \frac{\tilde{s}}{\lambda}\right)} \frac{1}{\Gamma\left(\frac{\tilde{s}}{\lambda}+1\right)} \exp \left(y \tilde{s}+O\left(\lambda^{-2}\right)\right) .
\end{aligned}
$$

Using the fact that the Laplace transform of the step function is expressed by the identity

$$
\int_{C} \frac{d s}{2 i \pi s} e^{s y}=\mathbf{1}(y>0)
$$

yields the large- $\lambda$ limit of the one-string contribution to the generating function as:

$$
Z\left(n_{s}=1, u\right) \underset{t \rightarrow \infty}{\sim}-\frac{1}{2} \int_{0}^{\infty} A i(y+u) d y .
$$

As in the continuum Lieb-Liniger model, assuming a Fredholm determinant structure for the cumulative distribution function of the rescaled free energy, as in the right-hand-side of Eq. 1, the one-string calculation yields the centering and scaling prescriptions that ensure that allow to write the left-hand side in terms of the parameters of the model only. Moreover, it yields the (opposite of the) trace of the kernel $B_{s}$, which supports the form of the kernel announced in Eq. 3. Indeed, the integer parameter organising the expansion of the Fredholm determinant in powers of the kernel is identical to the number of strings.

\section{$5 \quad$ Pfaffian structure of the generating function}

The odd mapping from rapidities to the string states of the log-gamma polymer, and the measuretheoretic factors worked out for parity-invariant string states, preserve much of the combinatoric structure of the generating function of the Lieb-Liniger model, based on the Pfaffian identities which we borrow [15], that allow to rewrite the inter-string products in in the factors $\Omega_{\mu}(\langle\mu \mid \mu\rangle)^{-1}$ terms of Pfaffians of antisymmetric matrices defined as $\operatorname{Pf}\left(\left(X_{i}-X_{j}\right)\left(X_{i}+X_{j}\right)^{-1}\right)$ from a vector $X$ with $n_{s}=2 N+M$ components containing linear combinations the parameters describing the strings (see Eqs 111-115 of [15]) The formal string expansion in terms of integer moments is therefore a modification of the formula 136 in Section 6 of [15], where the parameters $\left(k_{i}\right)_{1 \leq i \leq n_{s}}$ have been rescaled by a factor of $\bar{c}$, which is consistent with the replica result based on the overlap with the parity-invariant states:

$$
\begin{aligned}
Z^{m o m}\left(n_{s}, u\right)=\sum_{\left(m_{1}, \ldots, m_{2 n_{s}}\right) \in\left(\mathbf{N}^{*}\right)^{n_{s}}} \prod_{j=1}^{n_{s}} \int_{-\infty}^{+\infty} \frac{d k_{j}}{2 \pi} \prod_{q=1}^{m_{j}} \frac{-2}{2 i k_{j}+q} \Xi\left(m_{j}, \bar{c} k_{j}\right)^{\frac{t+1}{2}} e^{-\lambda m_{j} u} \\
\times \mathcal{P}\left(\left(k_{i}\right)_{1 \leq i \leq n_{s}},\left(m_{i}\right)_{1 \leq i \leq n_{s}}\right),
\end{aligned}
$$


where the Pfaffian factor $\mathcal{P}$ is evaluated on a matrix whose size is twice the number of strings, and the $i$-th string is specified by the real number $k_{i}$ and the integer number of particles $m_{i}$, so that the two terms in the upper-left block implement the selection rules of the localisation on parity invariant states:

$$
\begin{aligned}
& \mathcal{P}\left(\left(k_{i}\right)_{1 \leq i \leq n_{s}},\left(m_{i}\right)_{1 \leq i \leq n_{s}}\right)= \\
& \quad \operatorname{Pf}\left(\begin{array}{cc}
\frac{2 \pi}{2 i k_{i}} \delta\left(k_{i}+k_{j}\right)(-1)^{m_{i}} \delta_{m_{i}, m_{j}}+\frac{(2 \pi)^{2}}{4} \delta\left(k_{i}\right) \delta\left(k_{j}\right)(-1)^{\min \left(m_{i}, m_{j}\right)} \operatorname{sgn}\left(m_{i}-m_{j}\right) & -\frac{1}{2}(2 \pi) \delta\left(k_{j}\right) \\
\frac{1}{2}(2 \pi) \delta\left(k_{i}\right) & \frac{2 i k_{i}+m_{i}-2 i k_{j}-m_{j}}{2 i k_{i}+m_{i}+2 i k_{j}+m_{j}},
\end{array}\right)_{2 n_{s} \times 2 n_{s}}
\end{aligned}
$$

and the time-evolution is governed by the function of $m$ and $k$, which contains the parameter $\gamma$ of the distribution of Boltzmann weights (even though we dropped the symbol $\gamma$ from the notation):

$$
\Xi(m, k)=\frac{\Gamma\left(-\frac{m}{2}+\frac{\gamma}{2}-i k\right) \Gamma\left(-\frac{m}{2}+\frac{\gamma}{2}+i k\right)}{\Gamma\left(\frac{m}{2}+\frac{\gamma}{2}-i k\right) \Gamma\left(\frac{m}{2}+\frac{\gamma}{2}+i k\right)} .
$$

The compensation of the factors $\tau_{\mu}$ in both pairs of strings and strings with zero parameter $k$, ensured by the measure-theoretic factors, ensures that the only modification of the Lieb-Liniger is carried by the time-dependent factors (with a shift of time induced by the $\theta_{\mu}$ factors in the overlaps and norms of Bethe eigenstate labeled by $\mu$ ). The one-string constribution can be seen to reproduced the result derived above, as the constribution of the Pfaffian factor just consists of the upper-right entry of the matrix, enforcing the parity-invariant constraint on the string. Again borrowing from [15], we can represent each of the rational fractions in the string parameters entering the Pfaffian as a double integral of an exponential function, with a constraint, starting from the identity:

$$
\frac{2 i k_{i}+\lambda m_{i}-2 i k_{j}-\lambda m_{j}}{2 i k_{i}+\lambda m_{i}+2 i k_{j}+\lambda m_{j}}=\int_{0}^{\infty} d v_{i} \int_{0}^{\infty} d v_{j} \delta\left(v_{i}-v_{j}\right)\left(\partial_{v_{i}}-\partial_{v_{j}}\right) e^{-v_{i}\left(2 i k_{i}+\lambda m_{i}\right)-v_{j}\left(2 i k_{j}+\lambda m_{j}\right)} .
$$

Let us extract the exponential integrands from the matrix by use of the following multilinearity property of the Pfaffian, written in terms of square matrices $A$ and $B$ of size $n_{s}$, and vectors $U$ and $V$ with $n_{s}$ components,

$$
\operatorname{Pf}\left(\begin{array}{cc}
A_{i j} & \lambda_{i} U_{i} V_{j} \\
-\lambda_{j} U_{j} V_{i} & \lambda_{i} \lambda_{j} B_{i j}
\end{array}\right)_{2 n_{s} \times 2 n_{s}}=\left(\prod_{j=1}^{n_{s}} \lambda_{j}\right) \operatorname{Pf}\left(\begin{array}{cc}
A_{i j} & U_{i} V_{j} \\
-U_{j} V_{i} & B_{i j}
\end{array}\right)_{2 n_{s} \times 2 n_{s}}
$$

to obtain the $n_{s}$-string term as a multiple integral with all sums outside the Pfaffian:

$$
\begin{gathered}
Z^{m o m}\left(n_{s}, u\right)=\sum_{\{m\} \in\left(\mathbf{N}^{*}\right)^{n_{s}}} \prod_{j=1}^{n_{s}} \int_{v_{j} \geq 0} \int_{-\infty}^{+\infty} \frac{d k_{j}}{2 \pi} e^{-\lambda m_{j} u-v_{j}\left(2 i k_{j}+\lambda m_{j}\right)} \prod_{q=1}^{m_{j}} \frac{-2}{2 i k_{j} / \lambda+q} \Xi\left(m_{j}, k_{j} / \lambda\right)^{\frac{t+1}{2}} \\
\operatorname{Pf}\left(\begin{array}{cc}
\frac{2 \pi}{2 i k_{i}} \delta\left(k_{i}+k_{j}\right)(-1)^{m_{i}} \delta_{m_{i}, m_{j}}+\frac{(2 \pi)^{2}}{4} \delta\left(k_{i}\right) \delta\left(k_{j}\right)(-1)^{\min \left(m_{i}, m_{j}\right)} \operatorname{sgn}\left(m_{i}-m_{j}\right) & \pi \delta\left(k_{i}\right) \delta\left(v_{j}\right) \\
-\pi \delta\left(k_{j}\right) \delta\left(v_{i}\right) & 2 \delta^{\prime}\left(v_{i}-v_{j}\right)
\end{array}\right)_{2 n_{s} \times 2 n_{s}} .
\end{gathered}
$$


Putting back all the sums into the Pfaffian by the multilinearity property we express the $n_{s}$-string term as the integral of a Pfaffian:

$$
g_{\lambda}^{\text {mom }}(s)=1+\sum_{n_{s} \geq 1} \frac{1}{n_{s} !} \prod_{j=1}^{n_{s}} \int_{v_{j} \geq 0} \operatorname{Pf}\left(\begin{array}{cc}
\mathcal{K}_{11}^{\text {mom }}\left(v_{i}, v_{j}\right) & \mathcal{K}_{12}^{\text {mom }}\left(v_{i}, v_{j}\right) \\
-\mathcal{K}_{12}^{\text {mom }}\left(v_{i}, v_{j}\right) & \mathcal{K}_{22}^{\text {mom }}\left(v_{i}, v_{j}\right)
\end{array}\right)_{2 n_{s} \times n_{s}}
$$

where the kernels entering the Pfaffian are expressed as formal sums, and apart from

$$
\mathcal{K}_{22}^{m o m}\left(v_{i}, v_{j}\right)=2 \delta^{\prime}\left(v_{i}-v_{j}\right)
$$

contain sums over the numbers of particles, and time dependent powers of the kernel $\Xi$

$$
\mathcal{K}_{12}^{m o m}\left(v_{i}, v_{j}\right)=-\pi \delta\left(v_{j}\right) \sum_{m_{i} \geq 1} \frac{(-2)^{m_{i}}}{m_{i} !} \Xi\left(0, m_{i}\right)^{\frac{t+1}{2}} e^{-\lambda m_{i}\left(s+v_{i}\right)}=\tilde{\mathcal{K}}\left(v_{i}\right) \delta\left(v_{j}\right)
$$

which is a rank-one operator $\left(\right.$ on $\left.L^{2}(\mathbf{R})\right)$, with

$$
\begin{gathered}
\tilde{\mathcal{K}}(v)=\pi \sum_{m \geq 1} \frac{(-2)^{m}}{m !} \Xi(0, m)^{\frac{t+1}{2}} e^{-\lambda m(s+v)} \\
\mathcal{K}_{11}^{m o m}\left(v_{i}, v_{j}\right)=Q_{1}^{m o m}\left(v_{i}, v_{j}\right)+Q_{2}^{m o m}\left(v_{i}, v_{j}\right),
\end{gathered}
$$

with the following two formal kernels, adapted to the two sectors on which the phase-space measure of the two-string states localises (respectively the sector with one pair of strings with identical numbers of particles, and the sector with two strings with independent of particles and purely imaginary rapidities):

$Q_{1}^{\text {mom }}\left(v_{i}, v_{j}\right)=\int_{k_{i}} \frac{\pi}{i k_{i}} \sum_{m_{i} \geq 1}(-1)^{m_{i}} \Xi\left(m_{i}, k_{i} / \lambda\right)^{\frac{t+1}{2}} \Xi\left(m_{i},-k_{i} / \lambda\right)^{\frac{t+1}{2}} e^{\left.-\lambda m_{i}\left(2 s+v_{i}+v_{j}\right)-2 i k_{j}\left(v_{j}-v_{i}\right)\right)} \prod_{q=1}^{m_{i}} \frac{4}{q^{2}+4 k_{i}^{2} / \lambda^{2}}$

$Q_{2}^{\text {mom }}\left(v_{i}, v_{j}\right)=\frac{(2 \pi)^{2}}{4} \sum_{m_{i} \geq 1} \sum_{m_{j} \geq 1} \frac{e^{-m_{i} \lambda\left(s+v_{i}\right)}}{m_{i} !} \frac{e^{-m_{j} \lambda\left(s+v_{j}\right)}}{m_{j} !} \Xi\left(m_{i}, 0\right)^{\frac{t+1}{2}} \Xi\left(m_{j}, 0\right)^{\frac{t+1}{2}}(-1)^{\min \left(m_{i}, m_{j}\right)} \operatorname{sgn}\left(m_{i}-m_{j}\right)$.

Applying the analytic continuation prescription to the above kernels should yield a structure whose large-time limit can be studied, yielding the higher-order string expansion of the distribution function of the rescaled free energy. Pushing the string expansion up to the second order in $n_{s}$, is high enough to make all the kernels appear. 


\section{The two-string contribution to the generating function at large time}

In the two-string sector there are contributions from a pair of strings with opposite momentum parameters and identical number of particles (which require the integration of the weighted localised measure on pairs of strings worked out in the previous section), and contributions from two strings with zero parameter $k$, and any numbers of particles. The fact that $\mathcal{K}_{12}$ is a rank-one operator allows use to make use of the Fredholm identities of Appendix G of [15], which yield

$$
\begin{aligned}
Z\left(n_{s}=2, s\right) & =-\operatorname{Tr}\left(\mathcal{K}_{11}^{m o m} \mathcal{K}_{22}^{m o m}\right)=\int_{v_{1}} \int_{v_{2}} \mathcal{K}_{11}^{m o m}\left(v_{1}, v_{2}\right) \mathcal{K}_{22}^{m o m}\left(v_{2}, v_{1}\right) \\
& =-2 \int_{v_{1}} \int_{v_{2}} \mathcal{K}_{11}^{m o m}\left(v_{1}, v_{2}\right) \delta^{\prime}\left(v_{2}-v_{1}\right)=+2 \int \mathcal{K}_{10}^{m o m}(v, v) d v,
\end{aligned}
$$

with

$$
\mathcal{K}_{10}^{m o m}\left(v_{1}, v_{2}\right)=\partial_{v_{1}} \mathcal{K}_{11}^{m o m}\left(v_{1}, v_{2}\right)=\partial_{v_{1}} Q_{1}^{\text {mom }}\left(v_{1}, v_{2}\right)+\partial_{v_{1}} Q_{2}^{m o m}\left(v_{1}, v_{2}\right)
$$

\subsection{One pair of strings with identical numbers of particles}

Let us denote by $m$ the number of particles in each strings. The product of $m$ rational fractions of the $k$ parameter can be put into a form more suitable to analytic continuation to complex values of $m$ :

$$
\begin{aligned}
\prod_{q=1}^{m} \frac{1}{4 k^{2} / \lambda^{2}+q^{2}} & =\prod_{q=1}^{m} \frac{1}{(q-2 i k / \lambda)(q+2 i k / \lambda)} \\
& =\frac{1}{(1-2 i k / \lambda)_{m}(1+2 i k / \lambda)_{m}}=\frac{\Gamma(1-2 i k / \lambda) \Gamma(1+2 i k / \lambda)}{\Gamma(1-2 i k / \lambda+m) \Gamma(1+2 i k / \lambda+m)} .
\end{aligned}
$$

where use has been been of the Pochhammer symbol. The analytic-continuation prescription of the kernel $Q_{1}^{\text {mom }}$ therefore yields an expression whose large- $\lambda$ limit (which is equivalent to the large-time limit because of the choice of $\lambda$ as a function of time we made in Eq. 86), can yield a $\lambda$-independent equivalent, once the change of variable $m^{\prime}=\lambda m$ has been performed:

$$
\begin{aligned}
Q_{1}\left(v_{1}, v_{2}\right)= & -\int_{-a+i \mathbf{R}} \frac{d m}{2 i \pi \sin (\pi m)} \int_{-\infty}^{\infty} d k_{1} \int_{-\infty}^{\infty} d k_{2} \delta\left(k_{1}+k_{2}\right) \frac{\pi}{i k_{1}} \Xi\left(m, k_{1} / \lambda\right)^{\frac{t+1}{2}} \Xi\left(m, k_{2} / \lambda\right)^{\frac{t+1}{2}} \\
& e^{-\lambda m\left(2 s+v_{1}+v_{2}\right)+2 i\left(k_{1} v_{1}+k_{2} v_{2}\right)} \frac{\Gamma\left(1+2 i k_{1} / \lambda\right) \Gamma\left(1+2 i k_{2} / \lambda\right)}{\Gamma\left(1-2 i k_{1} / \lambda+m\right) \Gamma\left(1+2 i k_{2} / \lambda+m\right)} \\
& =\int_{-a+i \mathbf{R}} \frac{d m^{\prime}}{2 i \pi \lambda \sin \left(\pi m^{\prime} / \lambda\right)} \int_{-\infty}^{\infty} d k_{1} \int_{-\infty}^{\infty} d k_{2} \delta\left(k_{1}+k_{2}\right) \frac{\pi}{i k_{1}} \Xi\left(m^{\prime} / \lambda, k_{1} / \lambda\right)^{\frac{t+1}{2}} \Xi\left(m^{\prime} / \lambda, k_{2} / \lambda\right)^{\frac{t+1}{2}} \\
& e^{-m^{\prime}\left(2 s+v_{1}+v_{2}\right)+2 i\left(k_{1} v_{1}+k_{2} v_{2}\right)} \frac{\Gamma\left(1+2 i k_{1} / \lambda\right) \Gamma\left(1+2 i k_{2} / \lambda\right)}{\Gamma\left(1-2 i k_{1} / \lambda+m^{\prime} / \lambda\right) \Gamma\left(1+2 i k_{2} / \lambda+m^{\prime} / \lambda\right)}
\end{aligned}
$$

To capture the large-time limit of the contribution of two strings of opposite momenta, we can again use the Laplace method which involves the expansion of the logarithm of $\Xi(m, k)$ around 
$(0,0)$. Since this logarithm is odd in $m$ and even in $k$ (see Eq. 93), we can write it in the form:

$$
\begin{aligned}
\Phi(m, k) & :=\log \Xi(m, k) \\
& =-2 \psi\left(\frac{\gamma}{2}\right) m+\psi^{\prime \prime}\left(\frac{\gamma}{2}\right) m k^{2}-\frac{1}{12} \psi^{\prime \prime}\left(\frac{\gamma}{2}\right) m^{3}+\sum_{p \geq 2} \sum_{q=0}^{p} \xi_{p, q} k^{2 q} m^{2 p+1-2 q} \\
& =:-2 \psi\left(\frac{\gamma}{2}\right) m+\tilde{\Phi}(m, k) .
\end{aligned}
$$

where the coefficients $\xi_{p, q}$ can be expressed in terms of derivatives of the digamma function at $\gamma / 2$, ensuring that contributions of the same order in $k$ and $m$ will play the same role in the large-time limit if both variables are scaled by the same power of the parameter $\lambda$ (see Eq. 114). The linear term in $m$ will be discarded in the expansion, as the shift in the free energy levels implies that $\tilde{\Phi}$ will be substituted to $\Phi$ in all the expressions (as $\Phi(m, 0)=2 J_{\gamma}(m)$, whose linear term in $m$ dictated the centering of the free energy in the one-string contribution). Let us use a Laplace representation in the expansion parameter $m$ :

$$
\tilde{\Xi}(m, k)^{t}=\int_{0}^{\infty} \Upsilon(t, k, u) e^{-m u} d u
$$

where $\Upsilon$ is an inverse Laplace transform, with the free energy shifted as prescribed in the pairedstring contribution:

$$
\Upsilon(t, k, u)=\int_{C} \frac{d z}{2 i \pi} \tilde{\Xi}(m, k)^{t} e^{z u} .
$$

The change of variable defined by $u=\lambda v$ yields

$$
\tilde{\Xi}(m, k)^{t}=\lambda \int_{0}^{\infty} \Upsilon(t, k, \lambda v) e^{-m \lambda v} d v=\lambda \int_{0}^{\infty} d v \int_{C} \frac{d z}{2 i \pi} \tilde{\Xi}(k, z)^{t} e^{z \lambda v-m \lambda v},
$$

and the change of variable $w=\lambda z$ puts the expression in a form suitable to a series expansion of the exponent $\tilde{\Phi}$ around zero:

$$
\tilde{\Xi}(m, k)^{t}=\int_{0}^{\infty} d v \int_{C} \frac{d w}{2 i \pi} \exp \left(t \tilde{\Phi}\left(\frac{w}{\lambda}, k\right)+v(w-\lambda m)\right) .
$$

The argument of the exponential function in the above expression has terms of order 0 and 1 in $m$. Let us insert the expansion 108 and take care of the cubic term in $m$ using the Airy function and the scaling $\psi^{\prime \prime}(\gamma / 2) t=-8 \lambda^{3}$ that was identified in Eq. 86 based on the one-string contribution:

$\tilde{\Xi}(m, k)^{\frac{t}{2}}=\int_{0}^{\infty} d v \int_{C} \frac{d w}{2 i \pi} \exp \left(v(w-\lambda m)-4 \lambda^{2} w k^{2}+\frac{w^{3}}{3}-4 \frac{\lambda^{3}}{\psi^{\prime \prime}\left(\frac{\gamma}{2}\right)} \sum_{p \geq 2} \sum_{q=0}^{p} \xi_{p, q} \lambda^{2 q-2 p-1} k^{2 q} w^{2 p+1-2 q}\right)$.

As we need to insert the value of the kernel at $\left(k / \lambda, m^{\prime} / \lambda\right)$ into the integrand of the expression of $Q_{1}$, Eq. 115, the higher-order terms with the are all be weighted by a power of $\lambda$ equal to the total 
degree of the correction:

$$
\begin{aligned}
& \tilde{\Xi}\left(\frac{m}{\lambda}, \frac{k}{\lambda}\right)^{t / 2}=\int_{0}^{\infty} d v \int_{C} \frac{d w}{2 i \pi} \exp \left(v w-v m-4 w k^{2}+\frac{w^{3}}{3}-4 \frac{\lambda^{3}}{\psi^{\prime \prime}\left(\frac{\gamma}{2}\right)} \sum_{p \geq 2} \lambda^{-(2 p+1)}\left(\sum_{q=0}^{p} \xi_{p, q} k^{2 q} w^{2 p+1-2 q}\right)\right) \\
& \quad=\int_{-\infty}^{\infty} d q \operatorname{Ai}(q) \int_{0}^{\infty} d v \int_{C} \frac{d w}{2 i \pi} \exp \left(v(w-m)+\left(q-4 k^{2}\right) w-\frac{4}{\psi^{\prime \prime}\left(\frac{\gamma}{2}\right)} \sum_{p \geq 2} \lambda^{2-2 p}\left(\sum_{q=0}^{p} \xi_{p, q} k^{2 q} w^{2 p+1-2 q}\right)\right) \\
& \quad=\int_{-\infty}^{\infty} d q \operatorname{Ai}(q) \int_{C} \frac{d w}{2 i \pi(w-m)} \exp \left(\left(q-4 k^{2}\right) w-\frac{4}{\psi^{\prime \prime}\left(\frac{\gamma}{2}\right)} \sum_{p \geq 2} \lambda^{2-2 p}\left(\sum_{q=0}^{p} \xi_{p, q} k^{2 q} w^{2 p+1-2 q}\right)\right) \\
& =\int_{-\infty}^{\infty} d q \operatorname{Ai}(q) \exp \left(\left(q-4 k^{2}\right) m-\frac{4}{\psi^{\prime \prime}\left(\frac{\gamma}{2}\right)} \sum_{p \geq 2} \lambda^{2-2 p}\left(\sum_{q=0}^{p} \xi_{p, q} k^{2 q} m^{2 p+1-2 q}\right)\right) \\
& \quad=\int_{-\infty}^{\infty} d q \operatorname{Ai}\left(q+4 k^{2}\right) \exp \left(q m+O\left(\lambda^{-2}\right)\right) .
\end{aligned}
$$

Going back to the large-time equivalent of the quantity $\mathcal{K}_{10}$, we insert these representations, integrate over the variables $k_{2}$ with the constraint, and shift the variables of the two Airy functions, and take the large- $\lambda$ limit of the Gamma-function factors and the saddle-point limit of the integral:

$$
\begin{aligned}
Q_{1}\left(v_{1}, v_{2}\right) \underset{\lambda \rightarrow \infty}{\sim} 2 \pi & \int_{-\infty}^{\infty} \frac{d k}{k} \int_{-\infty}^{\infty} d q \operatorname{Ai}\left(q+4 k^{2}\right) \int_{-\infty}^{\infty} d r \operatorname{Ai}\left(r+4 k^{2}\right) \\
& \times \int_{C} \frac{d m^{\prime}}{2 i \pi \lambda \sin \left(\pi m^{\prime} / \lambda\right)} \exp \left(\left(q+r-2 s-v_{1}-v_{2}\right) m^{\prime}+2 i k\left(v_{1}-v_{2}\right)+O\left(\lambda^{-2}\right)\right) \\
& \times \frac{\Gamma\left(1+2 i k_{1} / \lambda\right) \Gamma\left(1+2 i k_{2} / \lambda\right)}{\Gamma\left(1-2 i k_{1} / \lambda+m^{\prime} / \lambda\right) \Gamma\left(1+2 i k_{2} / \lambda+m^{\prime} / \lambda\right)} \\
\underset{\lambda \rightarrow \infty}{\sim} 2 \pi & \int_{-\infty}^{\infty} \frac{e^{2 i k\left(v_{1}-v_{2}\right)} d k}{k} \int_{-\infty}^{\infty} d q \operatorname{Ai}\left(q+s+v_{1}+4 k^{2}\right) \int_{-\infty}^{\infty} d r \operatorname{Ai}\left(r+s+v_{2}+4 k^{2}\right) \theta(q+r) .
\end{aligned}
$$

This expression is independent of the parameter of the log-gamma model, and reproduces the largetime limit of the corresponding kernel in the Lieb-Liniger model, were the time-evolution factor is determined by the energies of the string states in rapidity space rather than by the kernel $\Xi$ (see Eqs 142 and 182 of Section 7 [15]).

\subsection{Two parity-invariant strings with independent numbers of particles}

Analytic continuation to complex $m_{i}$ and $m_{j}$ is impractical for the zero-momentum sector. However, we can make use of the expressions in terms of Gamma functions for the overlaps (Eq. 81 in [15]). However, writing the fixed- $m$ contribution factor as a Laplace transform as we did in the previous sector:

$$
\tilde{\Xi}(m, 0)^{t}=\int_{0}^{\infty} \Upsilon(t, 0, u) e^{-m u} d u
$$


where $\Upsilon$ is an inverse Laplace transform, with the free energy shifted as prescribed in the pairedstring contribution:

$$
\Upsilon(t, 0, u)=\int_{C} \frac{d z}{2 i \pi} \tilde{\Xi}(z, 0)^{t} e^{z u} .
$$

The change of variable defined by $u=\lambda v$ yields

$$
\tilde{\Xi}(m, 0)^{t}=\lambda \int_{0}^{\infty} \Upsilon(t, 0, \lambda v) e^{-m \lambda v} d v=\lambda \int_{0}^{\infty} d v \int_{C} \frac{d z}{2 i \pi} \tilde{\Xi}(z, 0)^{t} e^{z \lambda v} e^{-m \lambda v},
$$

and the change of variable $w=\lambda z$ puts the expression in a form suitable to a series expansion of the factor $\tilde{\Xi}$ around zero:

$$
\tilde{\Xi}(m, 0)^{t}=\int_{0}^{\infty} d v \int_{C} \frac{d w}{2 i \pi} \tilde{\Xi}\left(\frac{w}{\lambda}, 0\right)^{t} e^{v(w-\lambda m)} .
$$

Let us insert the expansion of Eq. 108 with $k=0$, which, using the time-scaling of the parameter $\lambda$, makes the time-independent cubic term in the exponent appear, giving rise to a factor that can be represented using the Airy function, with corrections that scale as negative powers of $\lambda^{2}$, and contain all the dependence on the parameter $\gamma$ of the discrete polymer model:

$$
\begin{aligned}
\tilde{\Xi}(m, 0)^{t} & =\int_{0}^{\infty} d v \int_{C} \frac{d w}{2 i \pi} \int_{-\infty}^{\infty} d q \operatorname{Ai}(q) \exp \left(q w+t \sum_{l \geq 2} \xi_{2 l+1}\left(\frac{w}{\lambda}\right)^{2 l+1}\right) e^{v(w-\lambda m)}, \\
& =\int_{-\infty}^{\infty} d q \operatorname{Ai}(q) \int_{C} \frac{d w}{2 i \pi} \frac{1}{\lambda m-w} \exp \left(q w+t \sum_{l \geq 2} \xi_{2 l+1}\left(\frac{w}{\lambda}\right)^{2 l+1}\right) \\
& =\int_{-\infty}^{\infty} d q \operatorname{Ai}(q) \exp \left(\lambda m q+O\left(\lambda^{-2}\right)\right)
\end{aligned}
$$

For integers values of the expansion parameter in the replica approach, we therefore obtain the following moment formula for the contribution of zero-momentum strings (with $\tilde{\lambda}$ the value of $\lambda$ associated to time $(t+1) / 2)$ :

$$
\begin{aligned}
Q_{2}^{m o m}\left(v_{1}, v_{2}\right) & =\frac{(2 \pi)^{2}}{4} \int_{-\infty}^{\infty} d q \operatorname{Ai}\left(q+s+v_{1}\right) \int_{-\infty}^{\infty} d r \operatorname{Ai}\left(r+s+v_{2}\right) \\
& \sum_{m_{1} \geq 1} \sum_{m_{2} \geq 1} \frac{e^{-\tilde{\lambda} m_{1} q}}{m_{1} !} \frac{e^{-\tilde{\lambda} m_{2} r}}{m_{2} !}(-1)^{\min \left(m_{1}, m_{2}\right)} \operatorname{sgn}\left(m_{1}-m_{2}\right) e^{\tilde{\lambda}^{3}\left(R_{\gamma}\left(m_{1}\right)+R_{\gamma}\left(m_{2}\right)\right)} \\
& =\frac{(2 \pi)^{2}}{4} \int_{-\infty}^{\infty} d q \operatorname{Ai}\left(q+s+v_{1}\right) \int_{-\infty}^{\infty} d r \operatorname{Ai}\left(r+s+v_{2}\right) F\left(2 e^{-\tilde{\lambda} q}, 2 e^{-\tilde{\lambda} r}\right)\left(1+O\left(\tilde{\lambda}^{-2}\right)\right)
\end{aligned}
$$

As the function $F$ has been obtained through Borel transform and inverse Laplace transform, and expressed in terms of Bessel functions in Appendix F of [15] in terms of Bessel functions, we can conjecture that the analytically continued large-time limit (or the time-independent component of it) is captured by neglecting the corrections in the above formula in a saddle-point approximation (which is consistent with the expected Fredholm-determinant structure and the fact that these corrections were neglected when working out the one-string contribution), and taking the large-time 
limit worked out in 15$]$ :

$$
F\left(2 e^{-\tilde{\lambda} q}, 2 e^{-\tilde{\lambda} r}\right) \underset{\lambda \rightarrow \infty}{\sim} \theta(r+q)(\theta(r) \theta(-q)-\theta(-r) \theta(q))
$$

so that

$$
Q_{2}\left(v_{1}, v_{2}\right) \underset{\lambda \rightarrow \infty}{\sim} \frac{(2 \pi)^{2}}{4} \int_{-\infty}^{\infty} d q \operatorname{Ai}\left(q+s+v_{1}\right) \int_{-\infty}^{\infty} d r \operatorname{Ai}\left(r+s+v_{2}\right) \theta(r+q)(\theta(r) \theta(-q)-\theta(-r) \theta(q)),
$$

which together with the large-time limit of $Q_{1}$ and the Fredholm Pfaffian structure of the generating function, allows to reproduce the argument of Section 7 of [15] yielding the $\gamma$-independent limit of the two-string contribution to the generating function

$$
\lim _{t \rightarrow \infty} Z\left(n_{s}=2, u\right)=\int \operatorname{det}\left[B_{u}\left(x_{1}, x_{2}\right)\right] d x_{1} d x_{2},
$$

where $B_{u}$ is the kernel based on the Airy function announced in Eq. 3 (whose trace can be recognised in $\left.Z\left(n_{s}=1, u\right)\right)$, and to confirm through algebraic reasoning that at each order $n_{s}$ in the string expansion,

$$
Z\left(n_{s}, u\right) \underset{\lambda \rightarrow \infty}{\sim}(-1)^{n_{s}} \int_{\mathbf{R}^{n_{s}}}\left(\prod_{k=1}^{n_{s}} d x_{k}\right) \operatorname{det}\left[\theta\left(x_{i}\right) \operatorname{Ai}\left(x_{i}+x_{j}+u\right) \theta\left(x_{j}\right)\right]_{n_{s} \times n_{s}},
$$

which yields the large-time convergence of the distribution of the rescaled free energy to the GOE Tracy-Widom distribution.

\section{$7 \quad$ Numerical tests}

As the above derivation contains several conjectural steps, independent numerical checks are crucial to compare direct simulations at small system size to the predicted features of the large-time limit of the model. The low values of the parameter $\gamma$ are the ones for which the divergence problem of the moments is the most severe. We may therefore choose the value $\gamma=3$ for numerical evaluations, as in [47], where the first two cumulants where studied for that value in the fixed-end case, for $N=10^{4}$ samples, and $t=4,096$, with a period $L=1,000$ and the starting point of the polymer at $(x=0, t=0)$.

The non-universal ( $\gamma$-dependent) predicted value

$$
\lim _{t \rightarrow \infty}\left(\overline{\overline{-\log Z_{t}}}\right)=\psi\left(\frac{\gamma}{2}\right)
$$

appears as a horizontal asymptote on Fig. 1, where the 1.h.s. of Eq. 127 is plotted as a function of time at exponentially growing values.

The next cumulant, with a rescaling factor containing the contribution of the parameter $\gamma$, allows to compare numerical results to the variance of the GOE Tracy-Widom distribution:

$$
\lim _{t \rightarrow \infty} \operatorname{Var}\left(\frac{\log Z_{t}}{\left(\frac{-t}{8} \psi^{\prime \prime}\left(\frac{\gamma}{2}\right)\right)^{1 / 3}}\right) \simeq 1.60
$$




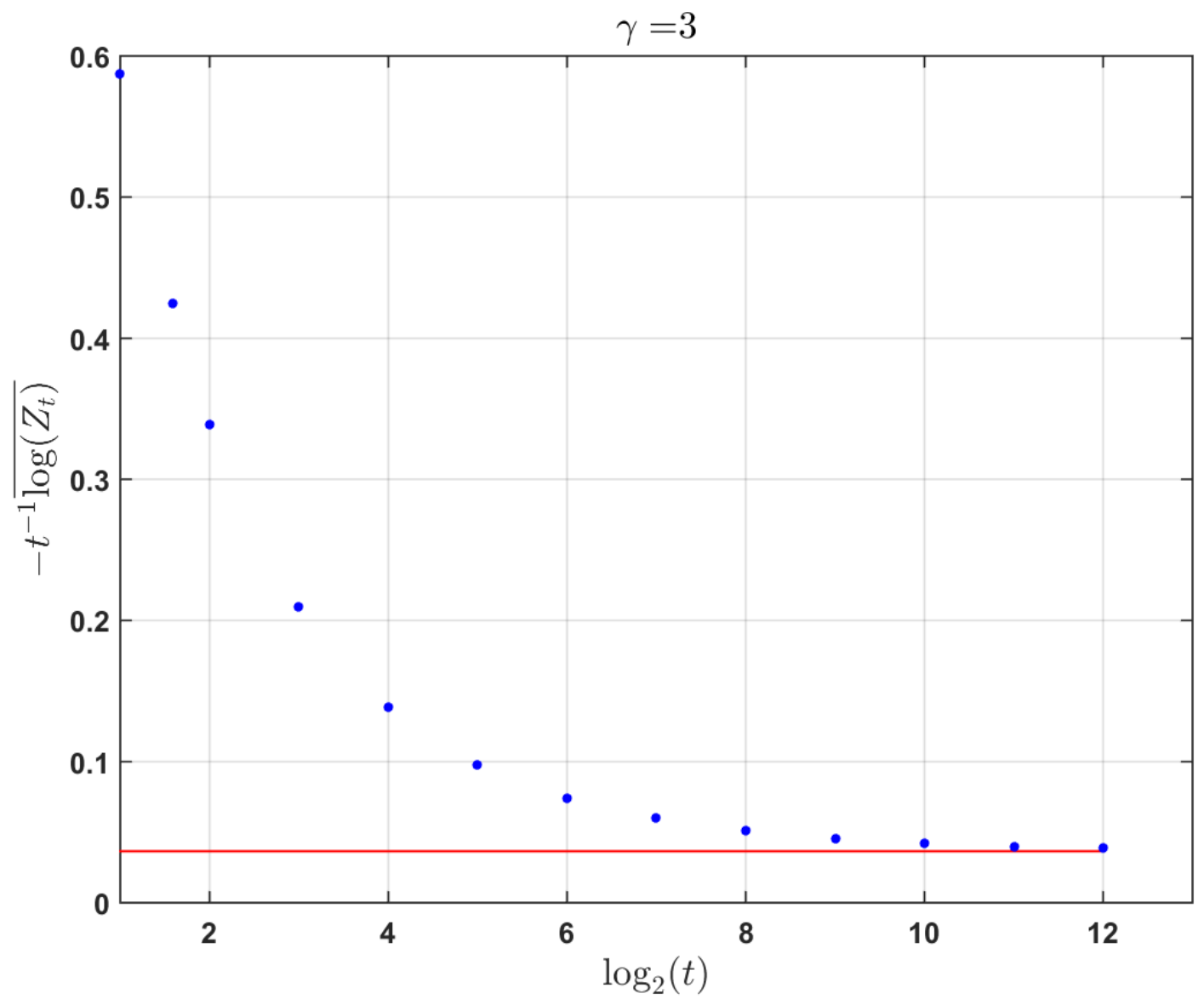

Figure 1: Convergence of the rescaled mean free energy (blue dots, with mean taken over $10^{4}$ samples) at $\gamma=3$ to the predicted value $\psi(\gamma / 2) \simeq 0.0365$ (red line). 


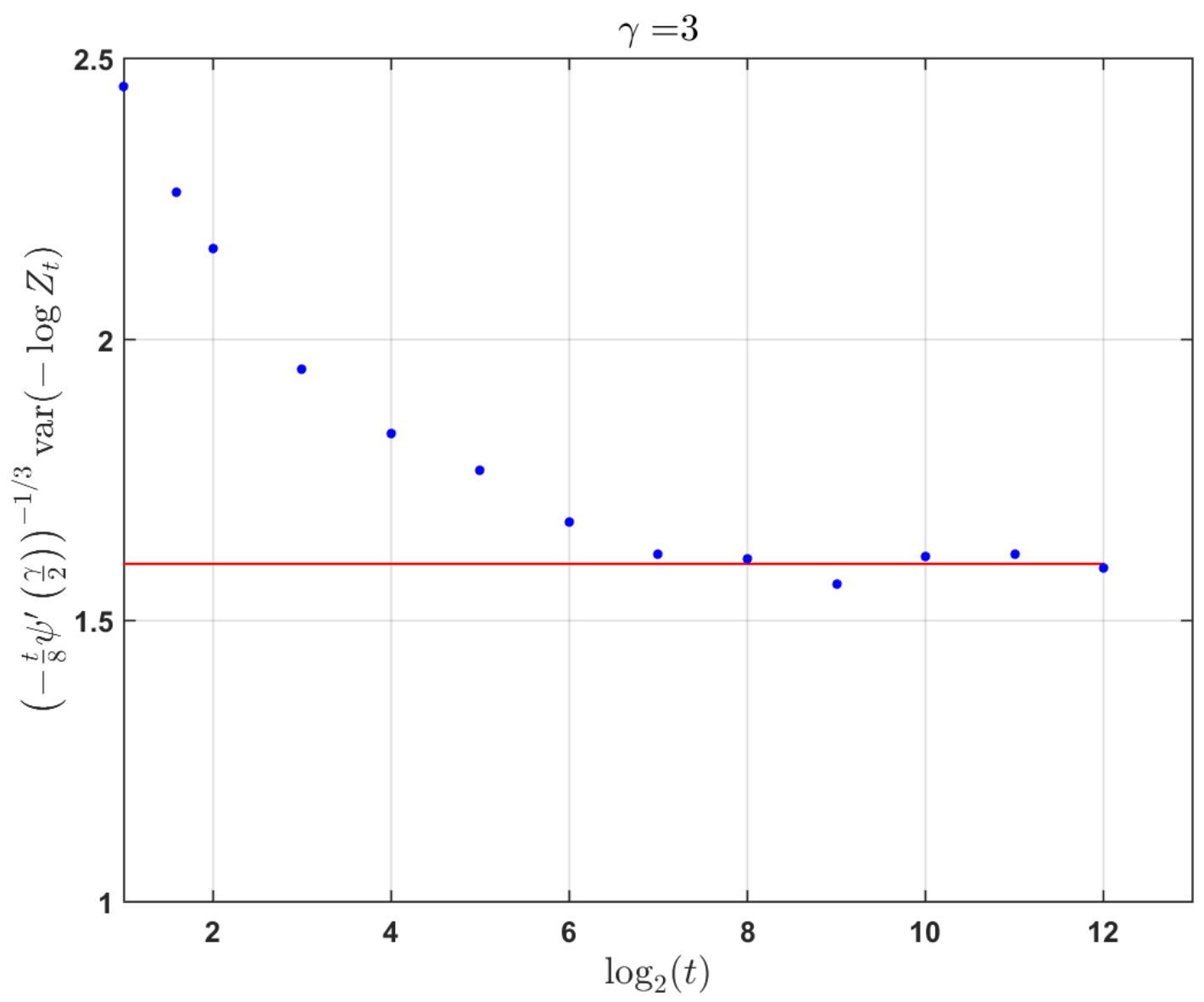

Figure 2: Convergence of the variance of the rescaled free energy (blue dots, with mean taken over $10^{4}$ samples) at $\gamma=3$ to the variance of the GOE probability distribution function (red line).

The 1.h.s. is plotted on Fig. 2, together with the predicted asymptote.

Moreover, the empirical distribution function of the rescaled free energy can be compared to the GOE prediction. Let us denote by $Z_{t}, k$ the partition function at time $t$ of the $k$-th sample. The empirical cumulative distribution function (e.c.d.f.) of the rescaled free energy

$$
\mathcal{P}_{N, t}(s)=\frac{1}{N} \sum_{k=1}^{N} \mathbf{1}\left(\frac{\log Z_{t, k}+\psi\left(\frac{\gamma}{2}\right) t}{\left(-\frac{t}{8} \psi^{\prime \prime}\left(\frac{\gamma}{2}\right)\right)^{\frac{1}{3}}}<s\right)
$$

at growing values of time against tabulated values of $F_{1}$ (using MATLAB files 4 available from http://www.wisdom.weizmann.ac.il/_nadler/Wishart_Ratio_Trace/TW_ratio.html for numerical evaluations of $F_{1}$ based on [51,52]). Curves quickly superpose and the empirical curve at time $t=4,096$ needs zooming to distinguish it from the universal prediction (see Fig. 3). The two curves

\footnotetext{
${ }^{4}$ in code available from

https://www.researchgate.net/publication/316454158_Simulation_of_the_log-gamma_polymer
} 
can be disentangled numerically by plotting the logarithm of the relative discrepancy

$$
\frac{\delta \mathcal{P}_{N, t}(s)}{\mathcal{P}_{N, t}(s)}=\frac{\left|\mathcal{P}_{N, t}(s)-F_{1}(s)\right|}{\mathcal{P}_{N, t}(s)},
$$

as a function of $s$ (see Fig. 4). In the domain $s>-4.4$ (i.e. for values of $s$ corresponding to more than $\left.F_{1}(s)>0.0022\right)$, the relative discrepancy is found to be less than one percent, and the average value of the decimal logarithm of the discrepancy over the domain is -5.58 .

Moreover, as the plotted numerical values of $F_{1}$ consist of $n_{F}=4097$ samples, the critical value of the absolute difference between the two curves in the two-sample Kolmogorov-Smirnov test at level $\alpha=0.001$ is

$$
\delta=1.95 \times \sqrt{\frac{N n_{F}}{N+n_{F}}}=0.0362,
$$

whereas the maximum difference between the predicted curve and the empirical curve at $t=4,096$ is found to be 0.0072 , which supports statistically the Tracy-Widom prediction. 


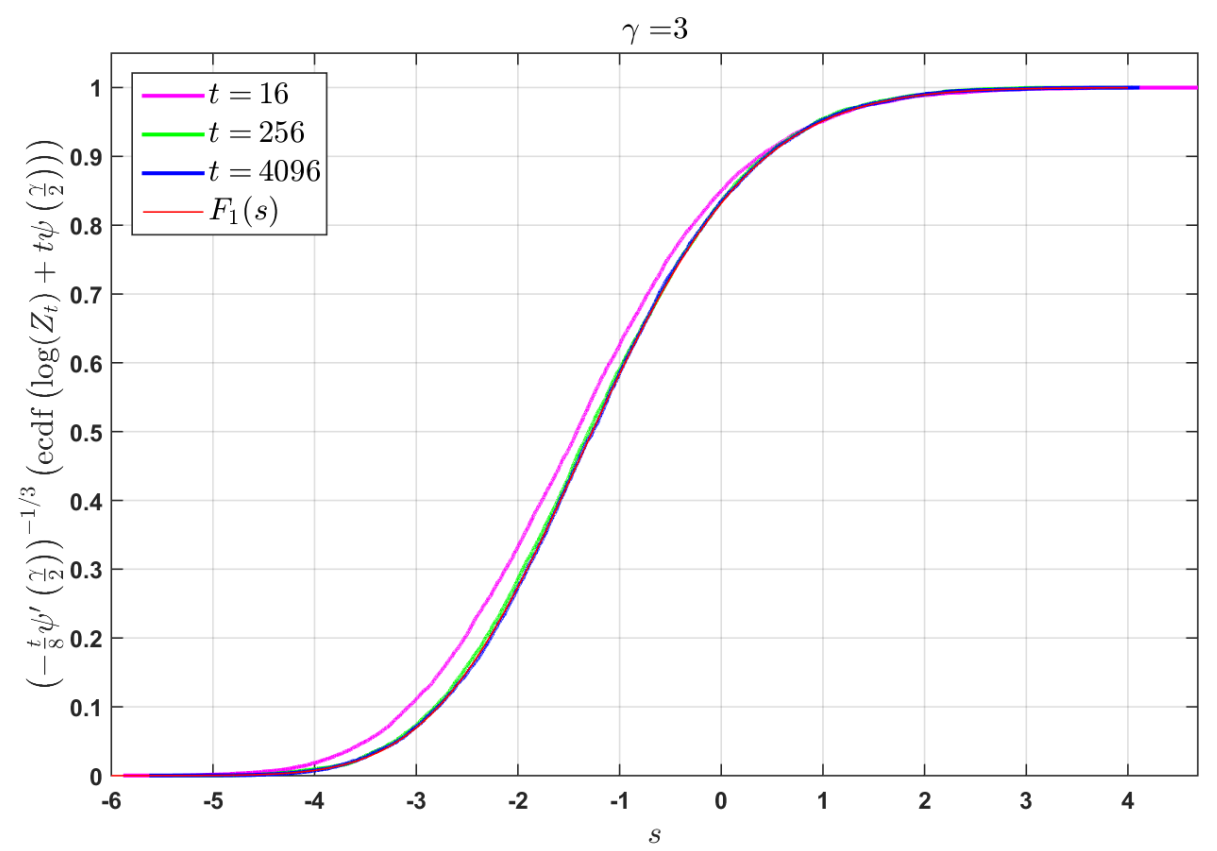

(a)

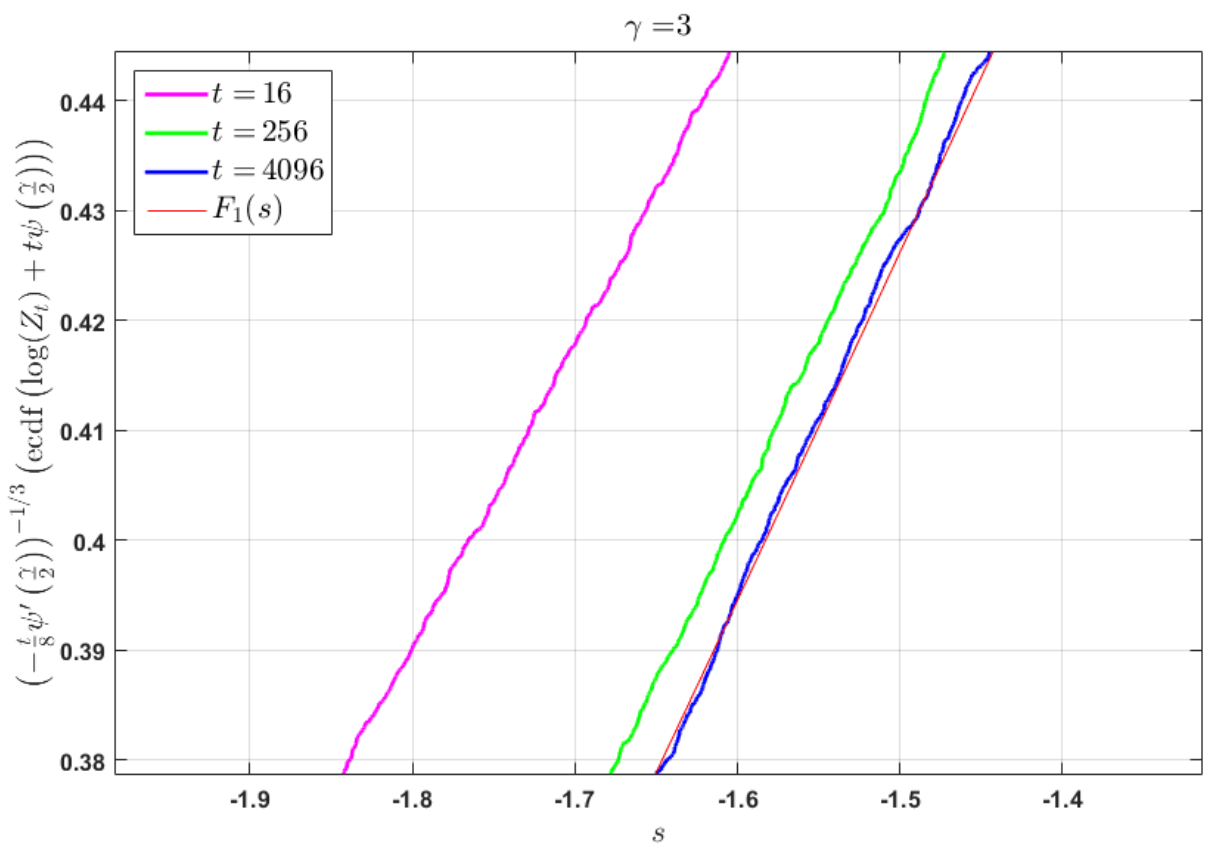

(b)

Figure 3: (a) Empirical cumulative distribution function of the rescaled free energy (Eq. 128) for $t=4,096$, based on $N=10^{4}$ samples, blue curve), and GOE Tracy-Widom function (red curve, based on 4,097 tabulated values for regularly spaced values of $s$ ). (b) Zoom around the value of $s$ corresponding to the asymptotic value the second cumulant. 


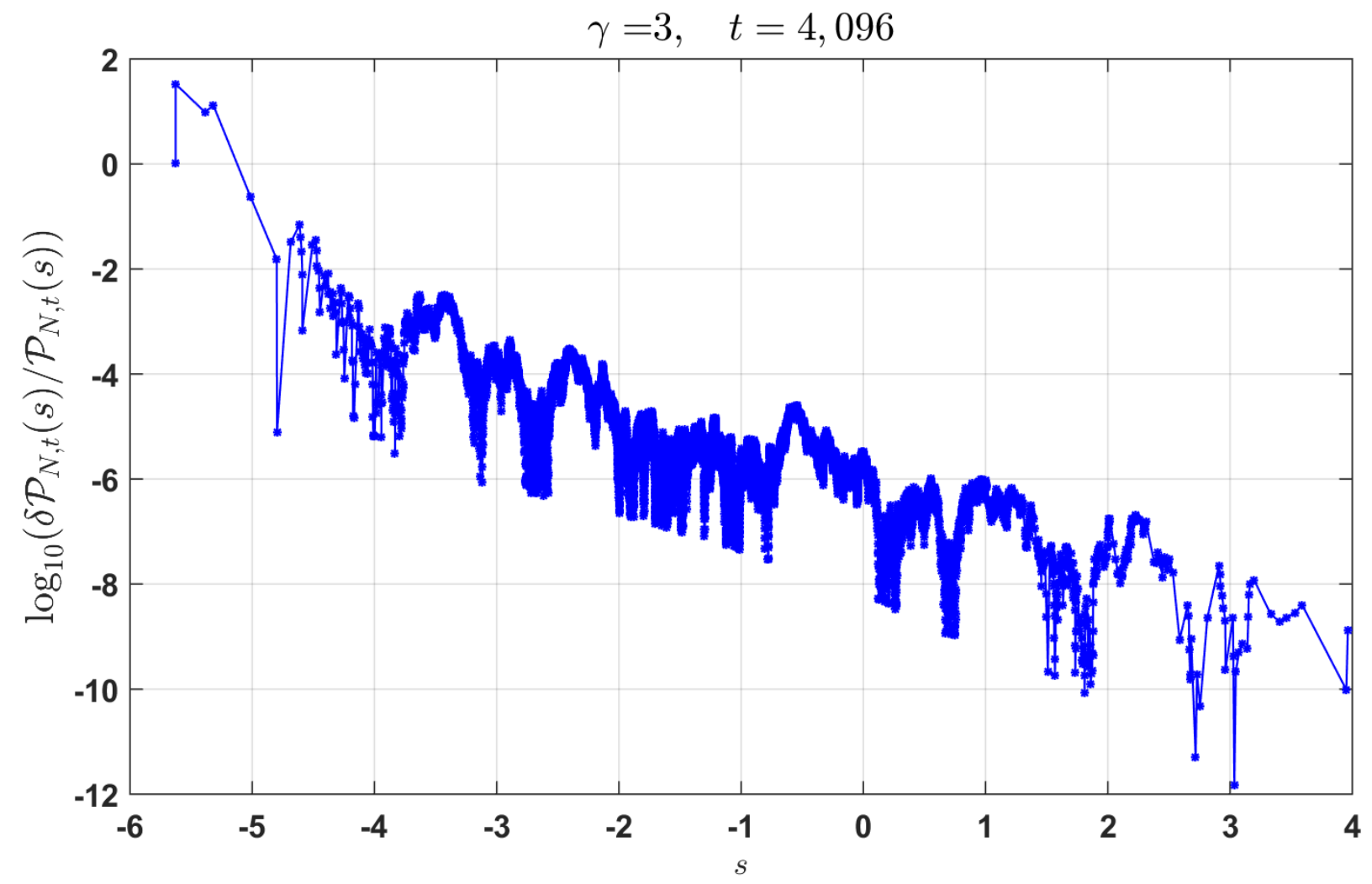

Figure 4: Logarithm of the relative discrepancy between the empirical cumulative distribution function of the rescaled free energy and the universal GOE prediction (Eq. 129), based on $N=10^{4}$ samples, at $t=4,096$. 


\section{Acknowledgments}

This work was supported by the Research Development Fund of Xi'an Jiaotong-Liverpool University (RDF-14-01-34).

\section{References}

[1] M. Kardar, G. Parisi, and Y.-C. Zhang, Dynamic Scaling of Growing Interfaces, Phys. Rev. Lett. 56, 889 (1986).

[2] T. Halpin-Healy and Y.-C. Zhang, Aspects of multidisciplinary statistical mechanics, Phys. Rep. 254(4-6):215-414 (1995).

[3] E.H. Lieb and W. Liniger, Exact analysis of an interacting Bose gas. I. The general solution and the ground state,Phys. Rev. 130(4), 1605 (1963).

[4] E. Brunet and B. Derrida, Ground state energy of a non-integer number of particles with delta attractive interactions, Physica A 279 395-407 (2000), |arXiv:cond-mat/0005355].

[5] E. Brunet and B. Derrida, Probability distribution of the free energy of a directed polymer in a random medium, Phys. Rev. E 61, 6789 (2000), ||arXiv:cond-mat/0005352].

[6] M. Gaudin, The Bethe Wavefunction, translation by J.-S. Caux, Cambridge University Press (2014).

[7] C.A. Tracy and H. Widom, Level-Spacing Distributions and the Airy Kernel, Comm. Math. Phys. 159151.

[8] P.L. Ferrari and H. Spohn, A determinantal formula for the GOE Tracy-Widom distribution, J. Phys. A: Math. Gen. bf38 L557-L561 (2005), | |arXiv:math-ph/0505012].

[9] P. Calabrese and J.-S. Caux, Correlation functions of the one-dimensional attractive Bose gas, Phys. Rev. Lett. 98(15), 150403 (2007).

[10] P. Calabrese and J.-S. Caux, Dynamics of the attractive 1D Bose gas: analytical treatment from integrability, J. Stat. Mech. P08032 (2007).

[11] P. Calabrese, P. Le Doussal and A. Rosso, Free-energy distribution of the directed polymer at high temperature, EPL (Europhysics Letters), 90(2), 20002 (2010), |arXiv:1002.4560|.

[12] V. Dotsenko, Bethe ansatz derivation of the Tracy-Widom distribution for one-dimensional directed polymers, EPL (Europhysics Letters), 90(2), 20003 (2010).

[13] V. Dotsenko and B. Klumov, Bethe ansatz solution for one-dimensional directed polymers in random media, J. Stat. Mech. 2010 (03), P03022.

[14] P. Calabrese and P. Le Doussal, Exact solution for the Kardar-Parisi-Zhang equation with flat initial conditions, Phys. Rev. Lett. 106(25), 250603 (2011), [arXiv:1104.1993]. 
[15] P. Le Doussal and P. Calabrese, The KPZ equation with flat initial condition and the directed polymer with one free end, J. Stat. Mech. (2012) P06001, |arXiv:1204.2607].

[16] T. Imamura and T. Sasamoto, Exact solution for the stationary Kardar-Parisi-Zhang equation, Phys. Rev. Lett. 108, 190603 (2012), ||arXiv:1111.4634|.

[17] T. Imamura and T. Sasamoto, Replica approach to the KPZ equation with half Brownian motion initial condition, J. Phys. A: Math. Theor. 44385001 (2011), ||arXiv:1105.4659|.

[18] T. Gueudré and P. Le Doussal, Directed polymer near a hard wall and KPZ equation in the half-space, $\mid$ arXiv:1208.5669|.

[19] V. Dotsenko, Two-point free energy distribution function in $(1+1)$ directed polymers, J. Phys. A 46355001 (2013)

[20] V. Dotsenko, Replica Bethe ansatz derivation of the GOE Tracy-Widom distribution in onedimensional directed polymers with free boundary conditions, [arXiv:1209.3603].

[21] V. Dotsenko, Distribution function of the endpoint fluctuations of one-dimensional directed polymers in a random potential, J. Stat. Mech. 2013(02), P02012.

[22] T. Imamura, T. Sasamoto and H. Spohn, On the equal time two-point distribution of the onedimensional KPZ equation by replica, J. Phys. A 46.35 (2013) 355002.

[23] S. Prolhac and H. Spohn, The one-dimensional KPZ equation and the Airy process, J. Stat. Mech. 2011.03 (2011): P03020.

[24] S. Prolhac and H. Spohn, Two-point generating function of the free energy for a directed polymer in a random medium, Journal of Statistical Mechanics: Theory and Experiment 2011.01 (2011): P01031.

[25] S. Prolhac and H. Spohn, Height distribution of the Kardar-Parisi-Zhang equation with sharpwedge initial condition: Numerical evaluations, Physical Review E 84.1 (2011): 011119.

[26] S. Prolhac and H. Spohn, The propagator of the attractive delta-Bose gas in one dimension, J. Math.Phys. 52.12 (2011): 122106.

[27] T. Sasamoto and H. Spohn, One-dimensional Kardar-Parisi-Zhang equation: an exact solution and its universality, Phys. Rev. Lett. 104, 230602 (2010), |arXiv:1002.1883].

[28] G. Amir, I. Corwin and, J. Quastel, Probability distribution of the free energy of the continuum directed random polymer in 1+ 1 dimensions, Comm. Pure Appl. Math 64, 466 (2011), |arXiv:1003.0443|.

[29] I. Corwin, The Kardar-Parisi-Zhang equation and universality class, "arXiv:1106.1596].

[30] P. Le Doussal, Crossover from droplet to flat initial conditions in the KPZ equation from the replica Bethe Ansatz, |arXiv:1401.1081]. 
[31] P. Calabrese and P. Le Doussal, Interaction quench in a Lieb-Liniger model and the KPZ equation with flat initial conditions, J. Stat.Mech. 2014 (5), P05004, |arXiv:1402.1278].

[32] P. Calabrese, M. Kormos and P. Le Doussal, From the sine-Gordon field theory to the KardarParisi-Zhang growth equation, EPL 107 (2014) 10011 |arXiv:1405.2582|.

[33] T. Thiery, Analytical methods and field theory for disordered systems, PhD thesis, available online at https://tel.archives-ouvertes.fr/tel-01361896/document.

[34] A.M. Povolotsky, On the integrability of zero-range chipping models with factorized steady states, J. Phys. A 46.46 (2013) 465205.

[35] T. Thiery and P. Le Doussal, On integrable directed polymer models on the square lattice, J. Phys. A 48(46) 465001 (2015), [arXiv:1506.05006|.

[36] T. Thiery, Stationary measures for two dual families of finite and zero temperature models of directed polymers on the square lattice, [arXiv:1604.07995].

[37] T. Thiery and P. Le Doussal, Exact solution for a random walk in a time-dependent 1D random environment: the point-to-point Beta polymer, [arXiv:1605.07538].

[38] N. O'Connell and M. Yor, Brownian analogues of Burke's theorem, Stoc. Proc. Appl. 96.2 (2001) 285-304.

[39] I. Corwin and L. Petrov, Stochastic higher spin vertex models on the line, Comm. Math. Phys. 343(2), 651-700 (2016), |arXiv:1502.07374].

[40] I. Corwin, T. Seppäläinen and H. Shen, The strict-weak lattice polymer, J. Stat. Phys. 160.4 (2015): 1027-1053, |arXiv:1409.1794].

[41] G. Barraquand and I. Corwin, Random-walk in Beta-distributed random environment, Probability Theory and Related Fields, 1-60, (2016), |arXiv:1503.04117].

[42] T. Imamura and T. Sasamoto, Current moments of 1D ASEP by duality, J. Stat. Phys. 142 (2011), 5, 919-930, |arXiv:1011.4588|.

[43] A. Borodin, I. Corwin and T. Sasamoto, From duality to determinants for q-TASEP and ASEP, Annals of Probability (2014), Vol. 42, No. 6, 2314-2382, |arXiv:1207.5035].

[44] T. Seppäläinen, Scaling for a one-dimensional directed polymer with boundary conditions, Ann. Probab., 40:1 (2012), 19-73. ||arXiv:0911.2446].

[45] I. Corwin, N. O’Connell, T. Seppäläinen and N. Zygouras (2014), Tropical combinatorics and Whittaker functions, Duke Mathematical Journal, 163(3), 513-563. ||arXiv:1110.3489].

[46] A. Borodin, I. Corwin and D. Remenik, Log-gamma polymer free energy fluctuations via a Fredholm determinant identity, Comm. Math. Phys. 324(1) (2013), 215-232.

[47] T. Thiery and P. Le Doussal, Log-gamma directed polymer with fixed endpoints via the replica Bethe Ansatz, J. Stat. Mech. (2014) P10018, |arXiv:1406.5963|. 
[48] E. Bisi and N. Zygouras, Point-to-line polymers and orthogonal Whittaker functions, |arXiv:1703.07337].

[49] J. De Nardis, B. Wouters, M. Brockmann and J.-S. Caux, Solution for an interaction quench in the Lieb-Liniger Bose gas, J. Phys. A (2013) 46:465205, ||arXiv:1308.3250].

[50] V.E. Korepin, Calculation of norms of Bethe wave functions, Comm. Math. Phys 86(3), 391418 (1982).

[51] F. Bornemann, On the numerical evaluation of distributions in random matrix theory: A review with an invitation to experimental mathematics, Markov Processes and Related Fields, 16 (4): 803866, |arXiv:0904.1581.

[52] B. Nadler, On the distribution of the ratio of the largest eigenvalue to the trace of a Wishart matrix, Journal of Multivariate Analysis, 2011. 